\title{
Quantum Gravity from Causal Dynamical Triangulations: A Review
}

\author{
R. Loll \\ Institute for Mathematics, Astrophysics and Particle Physics, Radboud University \\ Heyendaalseweg 135, 6525 AJ Nijmegen, The Netherlands. \\ and \\ Perimeter Institute for Theoretical Physics, \\ 31 Caroline St N, Waterloo, Ontario N2L 2Y5, Canada.
}

\begin{abstract}
This topical review gives a comprehensive overview and assessment of recent results in Causal Dynamical Triangulations (CDT), a modern formulation of lattice gravity, whose aim is to obtain a theory of quantum gravity nonperturbatively from a scaling limit of the lattice-regularized theory. In this manifestly diffeomorphism-invariant approach one has direct, computational access to a Planckian spacetime regime, which is explored with the help of invariant quantum observables. During the last few years, there have been numerous new and important developments and insights concerning the theory's phase structure, the roles of time, causality, diffeomorphisms and global topology, the application of renormalization group methods and new observables. We will focus on these new results, primarily in four spacetime dimensions, and discuss some of their geometric and physical implications.
\end{abstract}




\section{Introduction}

CDT Quantum Gravity or CDT for short denotes a nonperturbative path integral approach to quantum gravity, based on Causal Dynamical Triangulations. These represent curved, Lorentzian spacetimes and play the role of regularized lattice configurations in this prominent version of modern lattice gravity. CDT's key idea of how to introduce causal, Lorentzian features into gravitational path integrals based on piecewise flat spaces - which up to that point had been primarily Euclidean in nature $\mathrm{1}^{1}$ - while rendering them amenable to computation, dates back to 1998 .

From the initial formulation of this idea in two [5] and subsequently higher spacetime dimensions [6,7] it took several years of conceptual and technical development until the first breakthrough results were obtained for the full theory in four dimensions. They demonstrated the emergence of an extended fourdimensional universe with semiclassical features on large scales in one of the phases of CDT quantum gravity [8,9], something not seen previously (and since) in models of this type. In addition, the phenomenon of dynamical dimensional reduction was revealed as a nonperturbative quantum signature of spacetime on Planckian scales [10,11], sparking numerous investigations of the spectral dimension in other approaches to quantum gravity [12].

The next important finding came in 2007, when it was shown that the expectation value of the global shape of the dynamically generated universe behaves like the shape of a classical de Sitter universe [13, 14]. This further strengthened the evidence for the existence of a meaningful classical limit, something that nonperturbative approaches to quantum gravity generally struggle to demonstrate. Following this, strong evidence was found in 2011 for the presence of secondorder phase transitions in the phase space of the statistical model underlying CDT [15, 16]. Higher-order transitions are a crucial prerequisite for the existence of a well-defined continuum limit of a given lattice theory, and a feature that so far has eluded similar models of four-dimensional quantum gravity.

The last comprehensive review of CDT quantum gravity, published in 2012 [17], covered detailed derivations and descriptions of the geometric and technical set-up of the formalism, including the Monte Carlo simulations, a description of the model's phase structure and all of the above-mentioned classic CDT results in four dimensions, additional material on CDT systems with and without matter in two and three dimensions, many of which are interesting in their own right, and an extensive bibliography.

The main aim of the present article is to review the major new developments

\footnotetext{
1 "Euclidean spacetimes" have a Riemannian, positive-definite metric, instead of the physical, Lorentzian signature, and are used in the gravitational path integrals of Dynamical Triangulations (DT), the Euclidean precursor of CDT [1,2], and of Quantum Regge Calculus [3, 4.
} 
in CDT quantum gravity that have occurred since then, focusing primarily on the four-dimensional theory, and the potential physical significance of the results obtained. The latter include (i) the discovery of a new line of phase transitions, presumed to be of higher order, and an associated new phase, (ii) the introduction of two new observables, the gravitational Wilson loops and the quantum Ricci curvature, (iii) studies on the influence of global topology, and (iv) an implementation of the renormalization group. In addition, considering everything that has been learned so far, we will also try to assess the specific features of CDT that underlie its success to date as a candidate theory of nonperturbative quantum gravity. As described in more detail in Sec. 4 below, a key distinguishing feature appears to be the treatment of diffeomorphisms.

\section{Portrait of CDT as a young theory}

In a nutshell, CDT aims to construct a theory of quantum gravity by taking a suitable scaling limit of a lattice theory, whose dynamics is given in terms of a regularized, nonperturbative path integral over geometric lattices that in a direct way approximate the curved spacetimes of classical general relativity. In complete analogy with lattice QCD, say, the path integral depends on a number of bare coupling constants, which appear in a lattice version of the gravitational action $^{2}$, and on a UV lattice cutoff $a$ representing the shortest length unit on the lattice. As the regulator (or "lattice spacing") $a$ is removed, $a \rightarrow 0$, one searches for continuum limits in the vicinity of a critical point, characterised by the divergence of a suitable correlation length in terms of lattice units. This will usually involve a fine-tuning and renormalization of the dimensionless bare couplings of the lattice model.

Unlike quantum chromodynamics, quantum gravity is a theory we presently know rather little about. As a classical field theory, gravity has completely different degrees of freedom, dynamics and symmetries than a gauge theory. It is therefore not clear a priori that the construction of a fundamental quantum theory of gravity as the continuum limit of a lattice formulation is technically or even in principle feasible, and leads to physically meaningful results. Remarkably, CDT provides a blueprint for how potentially troublesome conceptual issues like the implementation of a Wick rotation and of symmetries, unitarity, and the unboundedness of the gravitational action can be dealt with in a lattice regularization. With these ingredients in place, progress to date in the construction and analysis of the nonperturbative quantum theory has been very promising indeed.

\footnotetext{
${ }^{2}$ After fine-tuning the cosmological constant to its critical value, the phase diagram of CDT is two-dimensional, spanned by the inverse bare Newton constant and the so-called asymmetry parameter, c.f. Sec. 6 below.
} 
The properties of the CDT model have been explored extensively but not exhaustively, primarily with the help of Monte Carlo simulations. Despite the fact that the CDT path integral is defined for Lorentzian signature, these powerful numerical methods can be applied because of the presence of a well-defined Wick rotation, a rare occurrence in quantum gravity beyond perturbation theory. More concretely, the quantitative evaluation of a number of invariant quantum observables has established several milestones in the form of concrete evidence for the existence of second-order phase transitions, the emergence of classical geometry, and the applicability of renormalization group methods, none of which was obvious at the outset. As transpires from the above, the means employed in CDT to search for the holy grail of quantum gravity are rather conventional: they are anchored in quantum field theory, without using fundamental higher-dimensional excitations like strings, loops or branes, postulating supersymmetry, invoking gauge-gravity dualities or a dynamical principle unifying all of the fundamental interactions.

Whether the no-frills approach of CDT quantum gravity, or any alternative approaches 18 20 will turn out to be successful or dead ends, complementary or mutually exclusive, or whether they are collectively barking up the wrong tree(s), is impossible to decide in the absence of a reliable quantum gravity phenomenology and in view of the incompleteness of our current candidate theories. Also CDT, despite its achievements, is still incomplete, with both conceptual and computational challenges ahead. In terms of physics, these broadly speaking concern the nature and properties of the theory and its vacuum state on Planckian scales. For example, can CDT provide independent, corroborating evidence for "asymptotic safety" [21 23], or indications of some other form of nonperturbative UV completion? Can its physics be described in terms of (generalized) geometry or some other effective degrees of freedom?

While work is ongoing to improve the efficiency of the simulations and to speed up measurements in the vicinity of the phase transitions - issues that are largely CDT-specific - all questions regarding the physical content of the theory and the properties of its ground state ("quantum spacetime") must be phrased in terms of observables. The search for observables transcends any particular approach to quantum gravity and is key to understanding the physical implications of any given theory. Stated briefly, observables in this context are diffeomorphisminvariant quantities, which are operationally well-defined and computable in the nonperturbative sector of the theory. Just a few observables are currently known, see [17] for further details and Sec. 7] below for an update.

An essential feature of CDT quantum gravity is the fact that it comes with its own reality check in the form of a well-developed "computational lab", allowing in principle the evaluation of arbitrary quantum-gravitational observables, subject to the usual limitations in terms of computing power, lattice size, finite- 
size effects, lattice artefacts and numerical errors. In the context of full-fledged quantum gravity, CDT has played its part in demonstrating that numerical methods can yield important, nonperturbative information, which currently cannot be obtained by any other means. Far from being "just numerics", numerical simulations allow the systematic testing of theoretical conjectures, be it on the analytical form of an effective action for the Friedmann scale factor in "true cosmology" (c.f. Sec. 8 below), or on specific properties of the theory's ground state. In this way numerical results feed back into the construction of a coherent and comprehensive theoretical framework for quantum gravity.

At the same time, CDT has helped to move the discussion of "which is the correct theory?" away from comparing the properties of specific approaches and formalisms, which often appear irreconcilable in terms of their initial assumptions and ingredients, to a better defined and potentially more fruitful comparison of (the spectra of) observables. The discussions around the spectral dimension one example of such a quantum observable - illustrate that there is scope for unexpected coincidences between different approaches, which in turn can motivate further research.

This particular example also shows that in nonperturbative quantum gravity no exotic ingredients are needed to produce highly surprising results, which could not have been anticipated from the (semi-)classical theory. In the case at hand this is the phenomenon of continuous dynamical reduction of the dimension of spacetime from the classical value of 4 to (at or near) 2 as one approaches the Planck scale. The computation and comparison of quantum observables of this kind can be a way forward in quantum gravity, helping us to identify its universal properties, until we manage to establish a stronger link with observation or experiment. Of course, any candidate theory must be sufficiently developed to permit unambiguous calculations, preferably without freely adjustable parameters and ad hoc auxiliary assumptions.

At age twenty, counting from the initial idea of making the gravitational path integral Lorentzian again [5], CDT quantum gravity has established itself as a serious contender for a nonperturbative theory of quantum gravity, on the basis of a significant body of work and results, several of which are unique to this approach. Of course, it is up to the informed readers to weigh the achievements of CDT relative to those of other approaches, and relative to the overall goals and past setbacks in quantum gravity. In our view, the outlook of CDT quantum gravity is bright: there are already beautiful results, the approach has not hit any major roadblocks or dead ends, and - as illustrated by the developments described in this review - there is a steady pace of progress and a clear way forward, hopefully with equally interesting results awaiting us in the near future.

Since CDT is in essence a quantum field-theoretic approach, its results can most easily be compared to continuum results obtained with the help of functional 
renormalization group methods in a setting with asymptotic safety, which are usually formulated in terms of the (gauge-fixed) spacetime metric $g_{\mu \nu}(x)$. This has already happened for two observables: the spectral dimension 24, 25] and the so-called volume profile, defined as the (expectation value of the) volume of space as a function of cosmological time [26]. However, recalling an earlier remark, because of the very different ways in which gauge and physical degrees of freedom are treated in the continuum and on the lattice, it is virtually impossible to compare such derivations meaningfully at an intermediate stage, say, at the level of some coarse-grained version of the metric, and for quantities that are not observables.

CDT quantum gravity in its regularized, Wick-rotated form can be thought of as a particular type of statistical mechanical system, whose degrees of freedom are not spins or matter fields on a given, fixed lattice, but instead the parameters describing the metric properties of the lattice, viewed as a piecewise flat space, which is itself subject to dynamics. In dimension two, where both DT and CDT were studied first, they constitute statistical systems of random geometry, which are soluble exactly with a variety of analytical methods, including combinatorial, matrix model and transfer matrix methods [27,29]. This provides an additional perspective in the sense that one can try to import two-dimensional structural analyses and solution strategies into four-dimensional CDT. More generally, as illustrated by the construction of the quantum Ricci curvature in Sec. 7.2, CDT has overlap with issues discussed in the fields of discrete geometry and computational geometry, and may look to these subjects for inspiration, for example, in its search for observables.

\section{CDT path integral - the bare essentials}

Since the details of how the CDT path integral over triangulations is set up have been covered in [17] and other previous reviews and overviews (see, for example, [30 34]), we will confine ourselves to a summary of the essentials, while highlighting some issues which are of special importance. The central object of interest in CDT quantum gravity is the nonperturbative gravitational path integral. For pure gravity, the CDT path integral $Z^{\mathrm{CDT}}$ takes the form of a continuum limit of a regularized lattice expression. Schematically, we have

$$
Z^{\mathrm{CDT}}\left(G_{\mathrm{N}}, \Lambda\right)=\lim _{a \rightarrow 0} \sum_{T \in \mathcal{T}} \frac{1}{C(T)} \mathrm{e}^{i S^{\mathrm{CDT}}[T]},
$$

where the sum is taken over inequivalent Lorentzian triangulations $T$, assembled from two types of elementary four-simplices according to certain causal gluing rules. 
Each term in the sum contributes with an amplitude depending on a lattice discretization $S^{\mathrm{CDT}}$ of the continuum Einstein-Hilbert action

$$
S^{\mathrm{EH}}=\frac{1}{G_{\mathrm{N}}} \int d^{4} x \sqrt{-\operatorname{det} g}\left(R\left[g, \partial g, \partial^{2} g\right]-2 \Lambda\right),
$$

where $G_{\mathrm{N}}$ and $\Lambda$ are Newton's constant and the cosmological constant respectively, and $R$ denotes the Ricci scalar of the metric tensor $g$. The quantity $C(T)$ in eq. (1) is the number of elements in the automorphism group of the triangulation $T$. It is equal to 1 whenever $T$ does not possess any symmetries, as is almost always the case. The lattice version of the action $(2)$ is subject to the usual discretization ambiguities. The standard choice for $S^{\mathrm{CDT}}$ is the so-called Regge version of the gravitational action [17, 35], but this choice is in no way fundamental or "exact", in the same way as Regge calculus in four dimensions has no fundamental physical status, but is merely a discrete approximation of general relativity. Recall that lattice quantum gravity - like lattice QCD - requires a continuum limit, guaranteeing the existence of universal properties on all scales, including Planckian, which are independent of the arbitrary choices made as part of the regularized lattice set-up (for example, the types of building blocks, the detailed form of the discretized action, the path integral measure and the gluing rules).

The action $S^{\mathrm{CDT}}$, derived explicitly in 7,17, , has a simple form and is easy to compute for a given simplicial manifold $T$. It is a linear combination of several counting variables $\left\{N_{i}^{I}\right\}$, where $N_{i}^{I}(T)$ denotes the number of simplices of dimension $0 \leq i \leq 4$ contained in $T$. The index $I$ labels different subtypes. For instance, the four-simplices of CDT come in two different variants, $(3,2)$ and $(4,1)$, with corresponding counting variables $N_{4}^{(3,2)}$ and $N_{4}^{(4,1)}$ (see Fig. 1). Schematically, the action can be written as

$$
S^{\mathrm{CDT}}[T]=k_{b} \pi \sqrt{4 \alpha+1} N_{0}(T)+\mathcal{A}\left(\alpha, k_{b}, \lambda_{b}\right) N_{4}^{(4,1)}(T)+\mathcal{B}\left(\alpha, k_{b}, \lambda_{b}\right) N_{4}^{(3,2)}(T),
$$

where $\mathcal{A}$ and $\mathcal{B}$ are specific linear combinations of the bare inverse Newton constant $k_{b}$ and the bare cosmological constant $\lambda_{b}$, and $\alpha>0$ is a finite, fixed parameter describing the geodesic length $\ell_{t}$ of a time-like edge in terms of the geodesic length $\ell_{s} \equiv a$ of a space-like edge according to

$$
\ell_{s}^{2}=a^{2}, \quad \ell_{t}^{2}=-\alpha a^{2}, \quad \alpha>0,
$$

see [17] for further details ${ }^{3}$

A frequently asked question is whether using (a discretized version of) the Einstein-Hilbert action in the path integral (1) amounts to a truncation of the

\footnotetext{
${ }^{3}$ Compared with formula (60) in [17, we have dropped a term proportional to the Euler characteristic of the spacetime, because it plays no role in the path integral.
} 


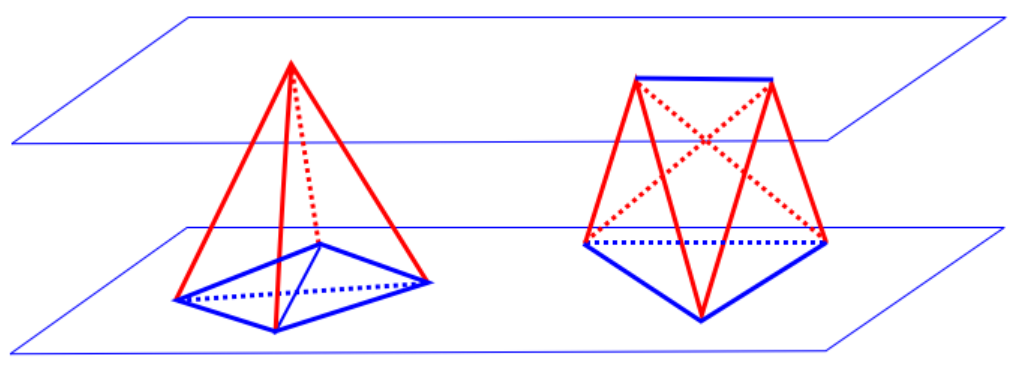

$(4,1)$

$(3,2)$

Figure 1: Regularized spacetimes in CDT quantum gravity are built from two elementary four-simplices, the (4,1)-simplex (left) and the (3,2)-simplex (right). They differ in their assignments of space- and time-like edges, drawn in blue and red respectively, see [17] for details.

theory. This is not the case. Since $S^{\mathrm{CDT}}$ is only a bare lattice action, not including curvature terms of quadratic or higher order in it does not imply a truncation, because - to use a continuum expression - such terms are generated during renormalization. More precisely, the full action governing the behaviour of the system at short distances receives contributions from both the bare action and the entropy of states (in a continuum language, the "path integral measure").

The difference it would make to include an $R^{2}$-term already in the bare action would be that the corresponding term in the full action would be associated with an additional, tuneable bare coupling parameter. One would be naturally led to consider such an extension of the parameter space of CDT if nothing interesting (for example, no higher-order phase transition) had been found in the version studied presently. Fortunately, the opposite is the case, which means that for the time being exploring CDT quantum gravity in its current form takes priority over possible extensions of the phase space.

Another way in which the action may be generalized is by including matter, accompanied by an additional sum over matter configurations for each spacetime configuration in the path integral. There are no specific technical difficulties in implementing this in dynamically triangulated quantum gravity, where gravitymatter models have been studied extensively in Euclidean-signature DT (see, for example, 2, 17]). Systems of CDT geometry and matter were investigated in the early days of CDT, and were centred on questions specific to two dimensions, like the existence of a $c=1$ barrier in CDT [36, 37] and whether matter critical exponents coincide with those on flat two-dimensional lattices [38, 39].

There are several reasons for why matter-coupled models have not yet been researched extensively in CDT in four dimensions. A key motivation for con- 
sidering matter coupling in four-dimensional Euclidean DT quantum gravity in the 1990s was the hope to find a continuous phase transition and an associated continuum theory after all [40], a hope that up to now has not been realized $4^{4}$. By contrast, four-dimensional CDT currently has plenty of interesting and nontrivial results even before matter is coupled, which are under active investigation. However, the issue of matter coupling is definitely interesting, and also presents new challenges. The primary one is to come up with physically interesting diffeomorphism-invariant matter observables, which are well-defined and finite in a nonperturbative regime where the underlying local spacetime geometry is very far from classical, as is the case in CDT. Technically, they need to be implementable and measurable on CDT lattices. Few such observables are currently known. On the conceptual side, as illustrated by studies of CDT coupled to a single massive particle [42,43], there are subtleties in relating Euclidean and Lorentzian results, which still need to be understood better.

Other important constructional aspects of the CDT path integral, discussed at length in [17], are its reflection positivity, virtually guaranteeing unitarity in the continuum limit, the existence of a rigorous Wick rotation which analytically continues the complex path integral (1) to a sum of real Boltzmann weights, and the implementation of Monte Carlo simulations. Apart from some remarks on the Wick rotation in Sec. 5 below, these will not be reviewed again here.

One can hardly overemphasize the importance of having well-developed quantitative tools available. CDT quantum gravity enables an interplay between theoretical considerations and numerical simulations: nonperturbative observables, suggested on theoretical grounds, can be implemented, tested and measured, and results from simulations can feed back into the theoretical modelling of Planckian physics, allowing an ever better understanding of what nonperturbative quantum gravity is about. This requires nontrivial expertise in the theoretical and practical aspects of Monte Carlo and lattice methods, and individuals trained in them. Many teams and researchers who worked or work with higher-dimensional dynamical triangulations have links with the lattice quantum field theory community, where these tools are commonplace, but this is by no means essential. Neither is there a need for investing in dedicated hardware, since the simulations are typically run on laptops or local computer clusters. In recent years, Markov chain Monte Carlo and other computer algorithms have gradually been making inroads, beyond lattice QCD and lattice gravity, into other quantum gravity approaches and more generally theoretical high-energy physics beyond the standard model (see $44-46]$ for some recent examples), and their appreciation appears to be on

\footnotetext{
${ }^{4}$ Note that integrating out matter fields in the path integral can lead to results equivalent to adding a higher-order curvature term in the gravitational action or including a nontrivial measure term, see [41] for a discussion in the context of four-dimensional Euclidean DT. All of these situations are associated with an extra coupling constant.
} 
the increase. Like in CDT, the emphasis is often on exploring the properties of given models and candidate theories, and performing "reality checks" of their viability, rather than improving the measuring accuracy of any given quantity by so many decimal places.

Broadly speaking, DT and CDT represent similar computational challenges in terms of algorithms, data storage and addressing. Computer code used in fourdimensional CDT in the early 2000s was adapted from previous DT versions, heavily used and tested over the previous decade. Computational efficiency of CDT has been improving steadily since. At the same time, a number of individuals and groups have independently written CDT code from scratch and run simulations 48 50], finding consistent results.5

\section{CDT geometry and diffeomorphism invariance}

CDT quantum gravity combines several key features in a unique way to produce its results. The two most important ones are

(i) the encoding of the geometric degrees of freedom of gravity, without introducing coordinates and their associated redundancy, and

(ii) the incorporation of Lorentzian features in the gravitational path integral, eq. (1), in a way that allows for its analytic continuation to a real partition function and the application of Monte Carlo methods.

This section spells out some details regarding point (i), while the next section summarizes the state of affairs with respect to point (ii). - The starting point for encoding the geometric degrees of freedom of gravity in a regularized way is Regge's proposal to describe "general relativity without coordinates" [35], where curved spacetimes are approximated by piecewise flat, simplicial manifolds, and a discrete analogue of the Einstein equations is derived. In this setting, a curved four-dimensional spacetime is described by its connectivity data (specifying which pairs of four-simplices share a three-dimensional face) and by the invariant lengths of the one-dimensional edges of the triangulation. All information about the intrinsic metric and curvature properties of a piecewise flat spacetime is expressible in terms of these data.

Note in passing that the space of piecewise flat spacetime geometries in CDT - serving as carrier space for the regularized gravitational path integral - is not identical to the one on which classical evolution is defined in [35]. The latter

\footnotetext{
${ }^{5} \mathrm{~A}$ publicly available version of the code, called CDT-plusplus, suitable for running on a variety of operating systems and cluster computing environments, will be released in the near future 47 .
} 
allows for continuous changes in the edge lengths of a given (topological) triangulation. This is by construction not possible in (C)DT, where all edge lengths are "frozen in" to one common length in DT, or two lengths (one for time-like, one for space-like edges) in CDT. It implies that CDT is not particularly wellsuited for describing classical continuous evolution, a purpose it also was never invented for. However, this is not an obstacle to formulating and computing the nonperturbative path integral, quite the contrary, as we will see. To summarize, (C)DT quantum gravity operates with a variable lattice topology ${ }^{6}$ and fixed edge lengths, while classical and quantum Regge calculus operate with fixed lattices and variable edge lengths, subject to triangle inequalities.

In concrete applications of CDT one often introduces discrete labels to refer to particular (sub-)simplices of a given triangulation, but this does not involve any coordinate system, and the ensuing relabeling redundancy is easily taken into account 7 One is of course free to introduce local coordinates in individual simplices or in a simply connected neighbourhood of a triangulation, as long as it does not contain any curvature singularities. However, this is redundant from a geometric point of view, and associated with the usual ambiguity of such a choice. The true strength of Regge's description is the absence of coordinates and their associated gauge freedom, together with a direct link of the edge length variables to intuitive geometric notions. The former is particularly important in quantum gravity beyond perturbation theory, where this freedom turns into the formidable difficulty of either having to gauge-fix or otherwise having to implement the four-dimensional diffeomorphism symmetry at the quantum level. CDT arguably makes optimal use of this strength in its construction of a nonperturbative gravitational path integral without any gauge redundancies 8

The pedagogical value of explaining curvature in terms of piecewise flat triangulations is illustrated nicely in 51]. More than that, when working in CDT and defining observables, the formulation forces one to think in terms of pure geometry and therefore true physics, very much in the spirit of Einstein's "rods and clocks", as opposed to resorting to some coordinate system. This latter feature is an asset of the formulation, even though it may appear unusual from the point of view of the classical theory, where differentiable manifolds and smooth metric fields provide powerful and convenient models of spacetime, and where choosing local coordinates is essential when performing concrete calculations. By

\footnotetext{
${ }^{6} \mathrm{By}$ this we mean different, inequivalent gluings of the flat building blocks, not the overall spacetime topology of the lattice, which in CDT is usually kept fixed.

${ }^{7}$ Basically, by dividing by $N$ ! for the case of $N$ labelled simplicial building blocks, leading to the path integral measure exhibited in (1) for unlabelled triangulations; see 17] for a more detailed discussion.

${ }^{8}$ The situation in quantum Regge calculus is less clear-cut, see 2 for a discussion and further references.
} 
contrast, in (C)DT quantum gravity the assignment of metric degrees of freedom is not smooth, there are no coordinates, and the four-dimensional diffeomorphism group simply does not act. This holds for the regularized path integral with finite UV-regulator $a$ and any continuum limit where $a \rightarrow 0$. In this sense, the folklore that "putting gravity on the lattice necessarily breaks diffeomorphism invariance" does not apply to CDT quantum gravity. As emphasized before, the treatment of diffeomorphisms in nonperturbative quantum gravity is in general highly nontrivial; by working directly on the quotient space of spacetime metrics modulo diffeomorphisms (or a suitable regularization thereof), this issue does not arise in CDT. Of course, any comparison of results and predictions of CDT, for example concerning its classical limit, with those coming from a more conventional smooth continuum formulation should be made in terms of appropriate geometric, coordinate-invariant observables. The fact that the DT approach can reproduce the results of a diffeomorphism-invariant continuum quantum field theory is well documented in the case of Liouville quantum gravity in two dimensions [52,53].

\section{Causal structure, Wick rotation, time and all that}

As highlighted in the introduction, the main motivation behind CDT was the search for a nonperturbative path integral formulation of gravity for physical spacetime geometries $g^{\text {lor }}$ of Lorentzian signature, schematically,

$$
\int_{\substack{\text { Lorentzian } \\ \text { spacetimes }}} D g^{\text {lor }} \mathrm{e}^{i S\left[g^{\text {lor }}\right]}, \quad \operatorname{signature}\left(g^{\text {lor }}\right)=(-+++) .
$$

This is different from so-called Euclidean quantum gravity, which uses a different starting point, namely, the path integral

$$
\int_{\substack{\text { Riemannian } \\ \text { "spacetimes" }}} D g^{\mathrm{eu}} \mathrm{e}^{-S\left[g^{\mathrm{eu}}\right]}, \quad \operatorname{signature}\left(g^{\mathrm{eu}}\right)=(++++),
$$

where Lorentzian spacetimes have been replaced in an ad hoc manner by Riemannian or (in physicists' parlance) Euclidean metric spaces $g^{\text {eu }}$. Since no Wick rotation $\urcorner^{9}$ is known that would map general curved, smooth Lorentzian metrics to Euclidean ones, or diffeomorphism equivalence classes of metrics of either signature into each other, there is no a priori physical justification for working with

\footnotetext{
${ }^{9}$ By Wick rotation we mean a suitable curved-space analogue of the flat-space prescription of substituting real time $t$ by imaginary time $\tau=i t$, for example through analytic continuation in the complex $t$-plane (see 54 for a discussion of why this is difficult to achieve).
} 


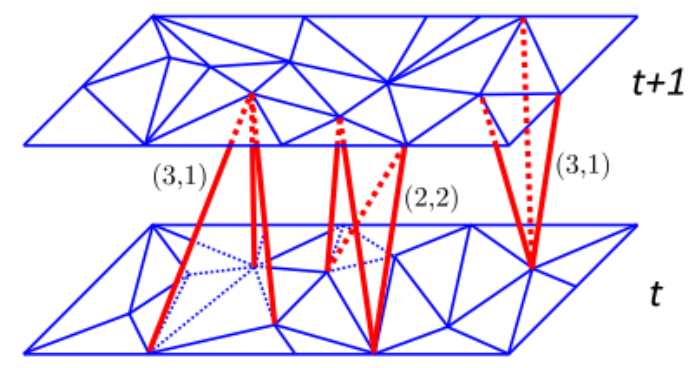

Figure 2: A "sandwich geometry" of topology $I \times{ }^{(2)} \Sigma$ for CDT in $2+1$ spacetime dimensions. The sandwich consists of a layer of three-simplices (tetrahedra) extrapolating between two adjacent spatial slices at times $t$ and $t+1$ made up of two-dimensional triangles. The space between the lower and upper triangulation is completely filled with tetrahedra, but for simplicity only three are shown here. (Space-like edges depicted in blue, time-like ones in red.)

Euclidean geometries, and no immediate reason why an associated nonperturbative path integral - if it exists - should agree with (or in some sense be equivalent to) any Lorentzian counterpart, or indeed have any physical interpretation at all.

The difficulty prior to the introduction of CDT was to find examples of Lorentzian nonperturbative path integrals that could be evaluated at all, either analytically or numerically, given that they are of the form of (weighted) sums or integrals over complex phase factors. One of the beautiful features of CDT quantum gravity is the existence of a well-defined Wick rotation that maps the piecewise flat spacetime configurations in the "sum over histories", eq. (1), to unique piecewise flat Riemannian spaces, while transforming the sum itself into a sum over real Boltzmann weights [6,7, 17]. It turns out that in two spacetime dimensions the analytically continued, real partition function $Z^{\mathrm{CDT}}$ can be evaluated analytically, which led to the first explicit demonstration that Lorentzian and Euclidean nonperturbative gravitational path integrals in general yield inequivalent results [5,29]. The same seems to be true in the physically relevant case of gravity in four dimensions, where the presence of the Wick rotation enables the evaluation of the CDT path integral and associated observables by Monte Carlo simulations, and thus an explicit comparison with corresponding DT results for Euclidean quantum gravity. Despite renewed efforts [41, 55, 56], there is still no evidence of higher-order phase transitions or semiclassical behaviour à la CDT in the latter.

The spacetimes summed over in the CDT path integral are assembled from two types of four-dimensional simplicial building blocks, as illustrated in Fig. 1. Each simplex can be thought of as a piece of flat Minkowski space, whose 


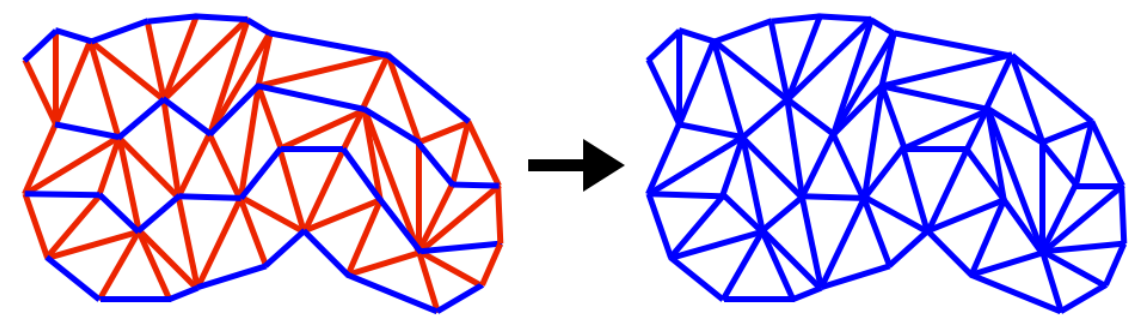

Figure 3: The layered structure of a two-dimensional CDT configuration, with space-like edges drawn in blue and time-like ones in red. Left: CDT geometry consisting of a stack of three sandwiches. The spatial geometries at discrete times $t$ are open chains consisting of a variable number $l_{t}>0$ of space-like edges. Right: the corresponding Euclidean geometry after Wick rotation. The underlying lattice structure is unchanged, but all time-like edges have been replaced by space-like ones.

geometric properties are fixed uniquely by the assignment of (squared) geodesic lengths to its 10 edges, given by $\ell_{s}^{2}=a^{2}$ for all space-like edges, and $\ell_{t}^{2}=-\alpha a^{2}$ for all time-like edges, for some $\alpha>0$. The gluing rules for these building blocks are such that the resulting spacetime configuration satisfies a lattice version of global hyperbolicity, in the sense that it has the form of a stack of "sandwich geometries". Each sandwich has topology $I \times{ }^{(3)} \Sigma$, where ${ }^{(3)} \Sigma$ denotes the fixed topology of a spatial slice, which in the situations studied so far is either a three-sphere $S^{3}$ or a three-torus $T^{3}$. Geometrically, a sandwich is a layer of thickness one in terms of the simplicial building blocks, with two purely space-like three-dimensional triangulations as initial and final boundaries (see Fig. 2 for an illustration of the analogous situation in one dimension less). One can attach a discrete proper time label $t$ to these sandwiches or, equivalently, to the spatial triangulations. In other words, each CDT spacetime can be seen as a sequence $t=0,1,2, \ldots, t_{\text {tot }}$ of spatial triangulations of topology ${ }^{(3)} \Sigma$, with $t_{\text {tot }}$ four-dimensional sandwich geometries extrapolating between pairs of adjacent spatial slices, to produce a simplicial Lorentzian manifold of total time extension $t_{t o t}$ with an initial and a final spatial boundary triangulation.

For the analogous but simpler case of CDT in two spacetime dimensions, Fig. 3 depicts a sequence of three sandwich layers. The elementary CDT building block in two dimensions is a Minkowskian triangle with one space-like and two time-like edges, and without loss of generality one can set $\alpha=1$. Wick-rotating in CDT amounts to analytically continuing $\alpha \mapsto-\alpha$ in the lower-half complex $\alpha$-plane [7, 17], which in the case at hand implies that all edges after the Wick rotation have equal length $\ell^{2}=a^{2}$. The resulting Euclidean triangulated curved space is shown in Fig. 3 on the right. Note that the triangulations cannot be 
represented isometrically in the plane because of their nonvanishing curvature assignments, associated with nonvanishing deficit angles at the vertices. Unlike what is usually done (including in Fig. 2 above), we have chosen deliberately to not depict the spatial universes by straight horizontal lines. The latter is a convenient, but ultimately arbitrary and potentially misleading choice, because it (wrongly) suggests an absence of extrinsic curvature of the spatial slices.

The layered structure is an important element of CDT and allows the straightforward implementation of a key feature, which distinguishes CDT geometries (before and after Wick rotation) from Euclidean DT geometries, namely, the absence of so-called "baby universes". These are violations of the local light cone (i.e. causal) structure, like those associated with branching points, where the spatial universe changes topology by branching out into two or more disconnected components. Such branchings are by definition disallowed in CDT, but they are generically present in DT, no matter what notion of time one superimposes on the Euclidean triangulations (for illustration, see for example [57, 58]). Although such violations of the causal structure are not allowed in the classical theory, there is no a priori reason to not permit them in path integral configurations. A major insight gained from CDT quantum gravity is that insisting on a well-behaved causal structure at this level appears to be essential for obtaining a nonperturbative quantum theory with a good classical limit. Note that analogous branchings of the geometry in the spatial directions are still perfectly allowed, are not in conflict with the causal structure and will generically occur. It means that in CDT - just like in the classical theory - space and time directions are not equivalent or interchangeable by symmetry 10 The fact that this distinction persists after the Wick rotation is at the heart of why the Lorentzian path integral can be inequivalent to (and lie in a different universality class than) its Euclidean counterpart.

Let us take the opportunity to remark on some properties of CDT quantum gravity associated with causal properties. Firstly, as just noted, the regularized CDT configurations come with a well-defined causal structure on all scales, which appears to be necessary for the model to have a four-dimensional behaviour compatible with classicality. It does not imply that a spacetime that emerges in a suitable scaling limit from a superposition of such configurations will necessarily inherit this structure. This is a property that will have to be checked in each case. It should also be noted that the causal structure of CDT configurations is part of their Lorentzian metric structure and as such is of course not fixed, but subject to quantum fluctuations, along with all other modes of geometry. Secondly,

\footnotetext{
${ }^{10}$ Note also that current investigations are far from a regime where one could make meaningful statements about the presence of local Lorentz invariance. This would require an (at least approximate) notion of local frames and the construction of a diffeomorphism-invariant quantum observable testing for Lorentz symmetry, objectives which at this stage seem out of reach.
} 
causal structure should not be confused with causality. The latter usually refers to the behaviour of matter or fields on a spacetime that is already endowed with a causal structure. The presence of the latter in the classical or quantum theory is a prerequisite for deciding whether or not excitations propagate causally.

The global, discrete time label $t$ and the associated family of three-dimensional spatial triangulations labelled by $t$ form a lattice substructure that is present in each CDT configuration. Note that $t$ is a parameter which can be used to (partly) specify the location of vertices, say, but is not related to any choice of gauge, because (i) there is no residual gauge invariance, as explained in Sec. 4 , and (ii) there are no coordinates in the first place, nor is it in general possible to introduce coordinate patches that extend beyond small neighbourhoods of pairs of adjacent simplices. Moreover, $t$ has an invariant geometric meaning on the piecewise flat CDT geometries. For the case of open spatial boundaries, it measures the geodesic (link) distance to the initial spatial boundary of the spacetime, while for the case of cyclically identified boundaries - at least in a phase with de Sitter behaviour - it measures the geodesic distance to the beginning of the universe, reminiscent of the diffeomorphism-invariant notion of cosmological time in classical general relativity [59].

Whether or not the label $t$ can be related to some physical notion of time in a suitable continuum limit cannot be determined a priori. What has been established is that in the phases of CDT where a de Sitter behaviour for the spatial volume of the universe is found, a renormalized version of $t$ assumes the role of global, cosmological proper time [13,14]. Whether there exist observables, for example, suitable two-point functions, whose behaviour allows for an interpretation of $t$ in terms of a specific, more local notion of time is currently not known.

While it is true that the regularized CDT geometries possess a layered or stacked structure, as we have just explained, there are several reasons to believe that this is just a lattice artefact and has no implications for the continuum limit. Firstly, although the situation resembles superficially the preferred foliation present in Hořava-Lifshitz gravity [60], which is associated with a breaking of full diffeomorphism invariance to an invariance under foliation-preserving diffeomorphisms, the discrete layers of CDT do not break any diffeomorphism symmetry, because the formulation does not have this symmetry to start with. Secondly, the action used in CDT quantum gravity is just the standard Einstein action, although one could in principle try to implement an explicitly noncovariant action of Hořava-Lifshitz type, as has been done in three dimensions [61]. Thirdly, there is a generalized version of CDT quantum gravity, where a well-behaved causal structure is implemented in terms of suitable local gluing rules, without any preferred lattice foliation. It has been investigated extensively in three dimensions, reproducing several key results of standard CDT [62 64], including the emergence of a de Sitter-shaped universe, thereby supporting the conjecture that it lies in 

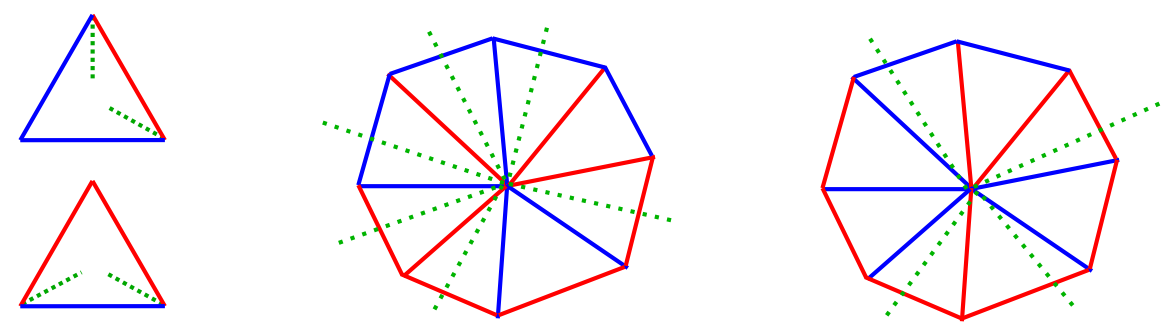

Figure 4: Left: the two elementary Minkowskian building blocks of CDT without preferred foliation in two dimensions; in standard CDT only the lower one is present. Dotted lines indicate light rays through the corner points. Centre: gluing these triangles together can result in local causality violations, like too many light cones meeting at a vertex, as shown. Right: at a causally well-behaved vertex, one crosses exactly four light-like directions when going around the vertex.

the same universality class. Since it touches on the fundamental issue of time in CDT, we will describe the construction and results of this model in some detail.

The primary motivation for studying "CDT without preferred foliation" or "locally causal DT" was to dissociate the causal structure of CDT from the preferred discrete foliation of the individual path integral histories, while staying in the purely geometric set-up of dynamical triangulations. The formulation introduced in [62] achieves this by allowing for additional simplicial building blocks without enlarging the set of allowed edge lengths, which are still given in terms of the relations (4). The gluing rules for these generalized elementary building blocks have to obey local causality conditions, to ensure that the local light cone structure is regular. (Fig. 4 illustrates the analogous situation in two dimensions, where the local causality conditions forbid the occurrence of a causality-violating configuration like the one depicted in the middle.) Unlike in standard CDT, a well-defined local causal structure is not guaranteed automatically, because the sandwich structure described earlier in this section is no longer present. Consequently, there is no preferred discrete lattice foliation and no preferred notion of time, although the CDT Wick rotation $\alpha \mapsto-\alpha$ is still applicable. Note that this also implies that there is no notion of performing the simulations for a fixed time extension $t_{\text {tot }}$.

For simulation-technical reasons the most convenient choice of the overall topology is not $S^{1} \times S^{2}$ with a cyclically identified time direction, but open boundary conditions where the two spatial two-spheres at the boundaries are compactified to points, resulting in an effective $S^{3}$-topology. Because the set of building blocks is enlarged and the Monte Carlo moves are more numerous and geometrically involved, the development of the simulation software turned out to be extremely complex. The system was simulated in a region of phase 
space spanned by the bare inverse Newton constant $k$ in the range $k \in[-1,1]$ and the asymmetry parameter $\alpha$ in the range $|\alpha| \in[0.5,1]$, and for fixed volumes $N_{3} \leq 160 k$. Just like standard CDT in three dimensions [65], the system exhibits a phase transition as a function of $k$, with a physical, "de Sitter-like" phase at low vertex density $N_{0} / N_{3}$ for $k<k^{c r i t} \approx 0.25$, and one at high vertex density above.

In order to compare with the results of standard CDT, one needs to reintroduce a notion of time, by referring only to intrinsic, geometric properties of the configurations. A natural choice, which can be used to associate a time variable with individual simplices and subsequently to define the notion of a spatial slice of constant time, is an average version of "link distance to the poles" of the three-sphere (see [63, 64] for details). Volume profiles as a function of this intrinsic time $\bar{t}$ were analyzed throughout phase space for $k<k^{c r i t}$ and found to both exhibit finite-size scaling (leading to a global Hausdorff dimension compatible with 3) and a high-precision matching with a $\cos ^{2}(\bar{t} /$ const)-shape expected for Euclidean three-dimensional de Sitter space. In addition, when approaching the upper kinematical range of $\alpha=0.5$ inside the physical phase, spacetime shows an increasing tendency to foliate dynamically, in the sense of resembling more and more that of a standard CDT configuration.

This provides additional nontrivial evidence that the preferred time slicing of Causal Dynamical Triangulations is not an essential element of its background structure, which is in line with the expectation formulated earlier. It also confirms the experience so far with dynamical geometric systems of DT-type, which suggests that universality is strong and there are very few universality classes. Because of the formidable difficulties that are already present in three dimensions, a similar analysis in four dimensions seems neither to be in reach nor to have high priority at this moment ${ }^{11}$ Note that the absence of the layered structure and preferred time of standard CDT implies that reflection positivity at the regularized level is not manifest in this generalized variant of CDT, which means that the status of unitarity is less clear-cut.

\section{$6 \quad$ Phase structure and phase transitions}

The regularized implementation in CDT of the Wick-rotated path integral for pure gravity has the form

$$
Z_{e u}^{\mathrm{CDT}}=\sum_{T \in \mathcal{T}} \frac{1}{C(T)} \mathrm{e}^{-S_{e u}^{\mathrm{CDT}}[T]} .
$$

\footnotetext{
${ }^{11}$ Perhaps more feasible, although less relevant physically, is the analytic solution of CDT without preferred slicing in two dimensions, to establish its equivalence or otherwise with standard CDT, which so far has not been achieved (see 66] for some numerical hints).
} 
The Euclidean action $S_{e u}^{\mathrm{CDT}}$ in the exponent is the result of analytically continuing the parameter $\alpha$ in the Lorentzian action (3) to $-\alpha$, according to the prescription outlined in Sec. 5 above ${ }^{12}$. The analytically continued action is purely imaginary, and combines with the imaginary $i$ in the exponent of the path integral (1) to yield the corresponding real Boltzmann weight in the partition function (7). A form of the Wick-rotated action that is convenient in simulations is obtained by substituting $\alpha$ by a new parameter $\Delta$, such that

$$
S_{\text {eu }}^{\mathrm{CDT}}[T]=-\left(\kappa_{0}+6 \Delta\right) N_{0}(T)+\kappa_{4}\left(N_{41}(T)+N_{32}(T)\right)+\Delta\left(2 N_{41}(T)+N_{32}(T)\right),
$$

where for ease of notation we have introduced $N_{41}:=N_{4}^{(4,1)}$ and $N_{32}:=N_{4}^{(3,2)}$, which we will use from now on ${ }^{13}$ To arrive at expression (8) from (the analytic continuation of) the action (3), one also has performed a linear redefinition of the bare couplings from $\left(k_{b}, \lambda_{b}\right)$ to $\left(\kappa_{0}, \kappa_{4}\right)$, see also ref. [17]. The so-called asymmetry parameter $\Delta$ depends on the parameter $\alpha$-describing the finite, relative scaling between the chosen geodesic lengths of time- and space-like links - in such a way that for equilateral triangulations (after Wick rotation) we have $\Delta=0$. However, it should be emphasized that neither this nor any other value of $\Delta$ is in any way distinguished on physical grounds a priori. At the level of the discretized action, different values correspond to building blocks which are more or less elongated in the time direction, but equally well suited to describing the (discretized) Einstein-Hilbert action. However, it has turned out that in the region of phase space associated with interesting physical behaviour, where contributions from the action and the entropy of states combine nonperturbatively, $\Delta i s$ an independent coupling constant, which may need to be fine-tuned in a scaling limit near one of CDT's critical points.

The coupling $\kappa_{0}$ in the action (8) is, up to a numerical constant, the inverse bare gravitational coupling, while $\kappa_{4}$ plays the role of bare cosmological constant. In CDT computer simulations, the lattice volume is (approximately) held fixed, to maximize efficiency. Since lattice volumes are necessarily finite, this is equivalent to fixing $\kappa_{4}$ to its (pseudo-)critical value, see [17 for an extended discussion. As usual in lattice field theory, one extrapolates to the continuum theory by studying the scaling properties of observables for a sequence of large and growing discrete lattice volumes, using so-called finite-size scaling techniques. Results reported for quantum observables, including in this review, usually refer to an extrapolation of measurements to infinite lattice volume, also known as the thermodynamic limit.

To summarize, the phase diagram of CDT quantum gravity is spanned by two bare coupling constants that can be varied independently, $\kappa_{0}$ and $\Delta$. In standard

\footnotetext{
${ }^{12}$ In order to satisfy the triangle inequalities, one needs $\alpha>7 / 2$ after the Wick rotation.

${ }^{13} \mathrm{In}$ some of the recent literature, the coupling $\kappa_{4}$ is redefined to $K_{4}:=\kappa_{4}+\Delta$, resulting in a rewriting of the action (8) to $S_{e u}^{\mathrm{CDT}}[T]=-\left(\kappa_{0}+6 \Delta\right) N_{0}(T)+K_{4}\left(N_{41}(T)+N_{32}(T)\right)+\Delta N_{41}(T)$.
} 


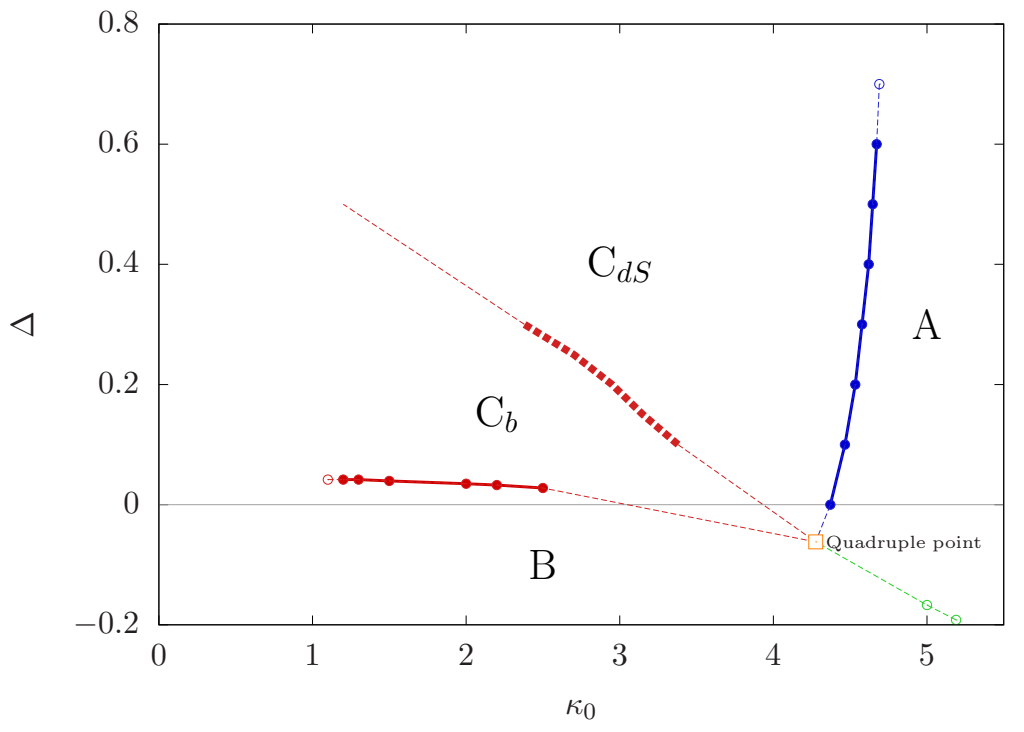

Figure 5: CDT phase diagram spanned by the bare couplings $\kappa_{0}$ and $\Delta$, consisting of the de Sitter phase $C_{d S}$, the bifurcation phase $C_{b}$, and two unphysical phases $A$ and $B$. (Fat dots and squares refer to actual measurements.)

simulations of CDT, two different prescriptions have been used to enforce an approximate lattice volume fixing, in terms of either the total number of foursimplices, $N_{4}=N_{41}+N_{32}$, or the number $N_{41}$ of $(4,1)$-simplices only. In physically interesting regions of phase space these lead to equivalent results, since at a given point $\left(\kappa_{0}, \Delta\right)$ the two four-simplex types occur approximately at a fixed ratio [17], where the ratio depends on the location in phase space. However, as will be mentioned below, the different prescriptions can influence the way in which phase transitions manifest themselves. To allow the volume to fluctuate in a small interval around a chosen target value $\bar{N}_{4}$ or $\bar{N}_{41}$, one includes a corresponding quadratic volume-fixing term of the form

$$
S_{f i x}^{\bar{N}_{4}}\left(N_{4}\right)=\varepsilon\left(N_{4}-\bar{N}_{4}\right)^{2}
$$

or

$$
S_{f i x}^{\bar{N}_{41}}\left(N_{41}\right)=\varepsilon\left(N_{41}-\bar{N}_{41}\right)^{2}
$$

in the bare action, where in either case $\varepsilon$ is an appropriately chosen small, positive parameter. This is needed because the update moves in the simulations are not volume-preserving.

The current understanding of the phase structure of CDT quantum gravity is illustrated by its phase diagram, depicted in Fig. $5{ }^{14}$ A new feature compared to

\footnotetext{
${ }^{14}$ The presence of a quadruple point in Fig. 5 is speculative. New evidence from measurements
} 
earlier discussions [17] is the phase transition line inside the physically interesting phase $C$, dividing it into a de Sitter phase $C_{d S}$ and a bifurcation phase $C_{b}$. It was discovered during investigations of the system with the help of the effective transfer matrix, to be described in more detail below. It had previously evaded attention because for the system sizes studied the global volume profile observable used to distinguish the different physical behaviours inside phases $A, B$ and $C$ is not sensitive to the $C_{b}-C_{d S}$ phase transition. The likely explanation is the nature of the bifurcation phase, whose distinguishing feature is a particular localized structure 67, 68 that does not affect the overall shape of the universe, at least not near the transition to the de Sitter phase. A similar observation holds for the order parameters that were used to locate the previously known transition lines $A-C$ and $B-C$ and determine their order [15, 16, 64, 69,70]. Although they can also be used for the $C_{b}-C_{d S}$ transition, the corresponding signals are much weaker than those of alternative order parameters that are attuned to more local features of the geometry [67,71].

In line with the usual logic of lattice quantum field theory, results on the order of CDT phase transitions are highly significant, because any transition point of second or higher order is a natural candidate for taking a scaling limit, and thus for the potential existence of a well-defined continuum limit independent of regularization details. CDT quantum gravity is currently unique in combining strong evidence for higher-order transition points and the applicability of Wilsonian renormalization group methods (see also Sec. 6.4 below) with strong evidence of the existence of a classical limit and the emergence of four-dimensional spacetime.

A first analysis of the order of CDT phase transitions was published in [69] on the basis of measurements for geometries of topology $S^{1} \times S^{3}$, time extension $t_{t o t}=80$ (cyclically identified) and fixed four-volume $N_{4}$. From the evolution in Monte Carlo time of the order parameter $N_{0} / N_{4}$ and its histogram at the $A-C_{d S}$ (former $A-C$ ) transition, it was concluded that this transition was most likely of first order. Repeating a similar analysis for the order parameter

$$
\operatorname{conj}(\Delta):=\left(-6 N_{0}+2 N_{41}+N_{32}\right)
$$

the quantity conjugate to $\Delta$ in the action $(8)$, at the $B-C_{b}$ (former $B-C$ ) transition yielded an inconclusive result. While the probability distribution for $\operatorname{conj}(\Delta) / N_{4}$ exhibited a double-peak structure, typically associated with two metastable states on either side of a first-order transition, the two peaks showed a tendency to approach each other for growing four-volume $N_{4}$, pointing to a second-order transition instead.

This motivated a careful study of the same system for larger volumes of up to $N_{4}=160 k$ 15, 16, repeating the histogram analysis, and supplementing it

for spatial tori, as described in more detail in Sec. 6.3, suggest there are two triple points instead. 


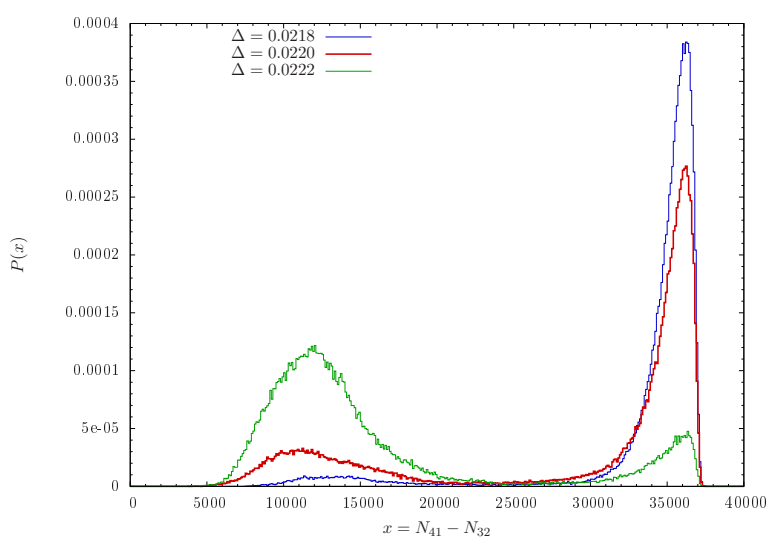

Figure 6: Probability distribution $P(x)$ of the order parameter $x=N_{41}-N_{32}$, closely related to (11), for fixed volume $N_{4}=40 k, t_{t o t}=80$ and $\kappa_{0}=2.2$. The measurements at three different $\Delta$-values near the critical point $\Delta_{c} \approx 0.0220$ on the $B-C$ transition line exhibit the double-peak structure characteristic for this volume fixing.

with independent criteria to establish the order of the phase transitions, namely, a measurement of the shift exponent (determined from the volume-dependence of the location of the maximum of the susceptibility) and an analysis of Binder cumulants. The results were mutually consistent and within measuring accuracy established the second-order nature of the $B-C$ transition ${ }^{15}$, while confirming the first-order character of the $A-C$ transition.

A more recent study of the $B-C$ transition [68] has confirmed its second-order status, and somewhat surprisingly found that the (potentially misleading) double peak in histograms simply disappears when $N_{41}$ instead of $N_{4}$ is kept fixed (see Figs. 6 and 7). A detailed quantitative analysis in the same work revealed that the origin of the two different behaviours has to do with the "entropy factor" $\mathcal{N}\left(N_{41}, N_{32}, N_{0}\right)$, counting the number of configurations for given values of the bulk variables $N_{41}, N_{32}$ and $N_{0}$, and the way in which it functionally depends on these variables. This confirms that the double-peak structure at the $B-C$ transition first reported in [69] in no way contradicts the conclusion of [15, 16 that this phase transition is of second order.

\subsection{Effective transfer matrix and new phase transition}

Along with the layered structure of standard CDT configurations and the associated discrete proper time label $t$ described in Sec. 5 above, CDT after the

\footnotetext{
${ }^{15}$ The same behaviour and a compatible numerical value for the shift exponent are found when using $t_{t o t}=2$ in an effective transfer matrix treatment 68.
} 


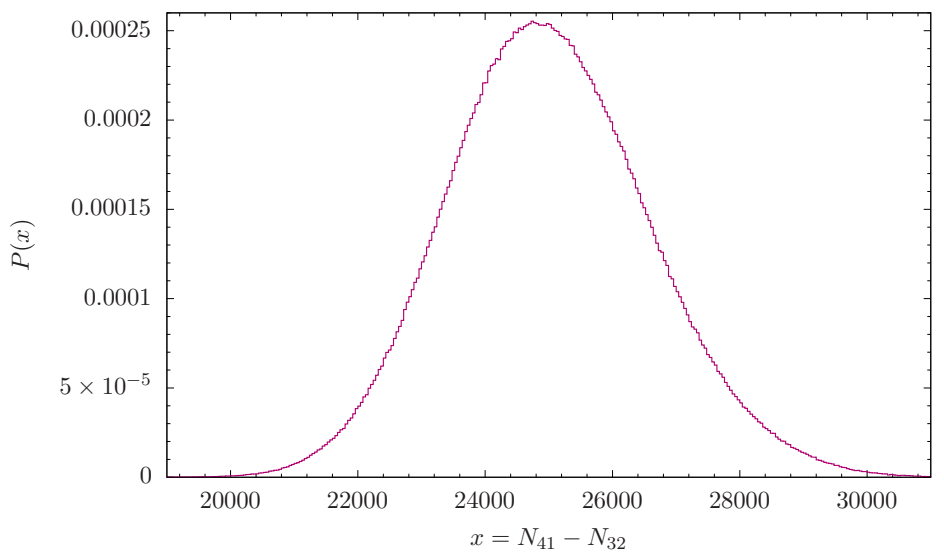

Figure 7: $\quad$ For fixed volume $N_{41}=33 k, t_{t o t}=80$ and $\kappa_{0}=2.2$, the measured probability distribution $P(x)$ of the order parameter $x=N_{41}-N_{32}$ close to the critical point $\Delta_{c} \approx 0.0220$ on the $B$-C transition line has only a single peak.

Wick rotation possesses a well-defined transfer matrix $\mathcal{M}$ [6, 7, 17]. Its matrix elements describe the amplitude of going from one three-geometry $T_{3}$ (a spatial three-dimensional simplicial manifold, usually of topology $S^{3}$ ) at time $t$ to another such geometry in the next time slice $t+1$,

$$
\left\langle T_{3}(t+1)|\mathcal{M}| T_{3}(t)\right\rangle=\sum_{T} \frac{1}{C(T)} \mathrm{e}^{-S_{e u}^{\mathrm{CDT}}[T]},
$$

where the sum is taken over all four-dimensional sandwich geometries $T$ with $\Delta t=$ 1 that extrapolate between the initial geometry $T_{3}(t)$ and the final geometry $T_{3}(t+$ 1 ). The transfer matrix acts on the infinite-dimensional vector space labelled by distinct three-dimensional triangulations $T_{3} \in \mathcal{T}_{3}$. Its elements $\left|T_{3}\right\rangle$ are equipped with the natural scalar product

$$
\left\langle T_{3} \mid T_{3}^{\prime}\right\rangle=\frac{1}{C\left(T_{3}\right)} \delta_{T_{3}, T_{3}^{\prime}}, \quad \sum_{T_{3}}\left|T_{3}\right\rangle C\left(T_{3}\right)\left\langle T_{3}\right|=\hat{\mathbf{1}}, \quad T_{3}, T_{3}^{\prime} \in \mathcal{T}_{3},
$$

where as before $C(T)$ denotes the size of the automorphism group of the triangulation $T$. Transition amplitudes between spatial three-geometries that are $t_{\text {tot }}$ time steps apart can be obtained by iterating the transfer matrix $t_{t o t}$ times, resulting in $\mathcal{M}^{\text {tot }}$. Note that the Hilbert space decomposes into subspaces spanned by three-geometries sharing the same discrete three-volume, namely, the number $n_{T}:=N_{3}(T)$ of tetrahedra of a given $T \in \mathcal{T}_{3}$. The number of three-dimensional simplicial manifolds with volume $n$ is finite and for large volume grows exponentially with $n+16$

\footnotetext{
${ }^{16}$ The notation $n$ is used in the literature both for the discrete three-volume of spatial slices and for twice the discrete three-volume, sometimes even in the same paper 72 . Note that in
} 
The idea of the effective transfer matrix $M$, introduced in [72], is the construction of a simpler quantity than $\mathcal{M}$ itself, which can nevertheless capture important information of the full system ${ }^{17}$ As will become clear below, this has proven a valuable strategy for describing the dynamics of the three-volume and its associated correlators, as well as for the analysis of CDT's phase structure. As mentioned earlier, it also led to the discovery of the $C_{b}-C_{d S}$ phase transition. The states $|n\rangle$ associated with the effective transfer matrix are labelled by the three-volume $n$ only, and can be thought of as arising from uniform probability distributions of states $\left|T_{3}\right\rangle$ sharing the same three-volume $n$. Recall that the threevolume as a function of proper time plays an important role in CDT, since its expectation value, the volume profile $\left\langle n_{t}\right\rangle$, in phase $C$ can be matched to that of a (Euclidean) de Sitter space, and the behaviour of the quantum fluctuations of the three-volume to those of a semiclassical minisuperspace treatment 13, 14, 74, 75.

It is entirely nontrivial that a reduced transfer matrix with matrix elements $\left\langle n_{t+1}|M| n_{t}\right\rangle$ should exist and can reproduce these results, but this is what has been found [72,75,77]. A big computational advantage of this formalism, wherever it applies, is the direct accessibility of its matrix elements in simulations, and the fact that it avoids time-consuming simulations of full-size CDT configurations with $t_{t o t} \approx 80$, in favour of measurements involving a small number of time slices. In [72], transfer matrix elements $\left\langle n_{t+1}|M| n_{t}\right\rangle$ were obtained from combining measurements of various probability distributions of pairs $\left(n_{1}, n_{2}\right)$ of three-volumes, for (cyclically identified) total times in the range $t_{\text {tot }} \in[2,6]$, at $\left(\kappa_{0}, \Delta\right)=(2.2,0.6)$, and using a novel, local volume fixing. Independent of the specific choices made and for a wide range of three-volumes the measured matrix elements turns out to be well described by the ansatz

$$
\left\langle n_{t+1}|M| n_{t}\right\rangle \propto \mathrm{e}^{-L_{\text {eff }}\left[n_{t}, n_{t+1}\right]},
$$

with the effective Lagrangian

$$
L_{\mathrm{eff}}[n, m]=\frac{1}{\Gamma} \frac{(n-m)^{2}}{(n+m)}+\mu\left(\frac{n+m}{2}\right)^{1 / 3}-\lambda\left(\frac{n+m}{2}\right) .
$$

One recognizes the functional form of $L_{\text {eff }}$ from the effective action for the threevolume reconstructed from the covariance matrix of spatial volume fluctuations around the semiclassical expectation value $\left\langle n_{t}\right\rangle$ in the full CDT simulations

terms of the former interpretation one has $2 n_{T}=N_{41}(T)$ for a configuration $T$ with compactified time, because each space-like tetrahedron in a slice of constant integer time is shared by two four-simplices of type $(4,1)$. Wherever the difference matters, for example, in some explicit fitting formulas for the effective action, readers are advised to consult the original papers.

${ }^{17}$ See 73 for an attempt to model the phase structure of CDT in terms of a one-dimensional lattice model whose transfer matrix resembles the effective transfer matrix discussed here. 
[13, 14, 74, It consists of a kinetic term $\propto 1 / \Gamma$, a three-dimensional scalar curvature term $\propto \mu$ and a cosmological constant term $\propto \lambda$, where the parameters $\Gamma, \mu$ and $\lambda$ depend on the couplings $\kappa_{0}$ and $\Delta$. Correction terms $\propto \mathcal{O}\left((n+m)^{-1 / 3}\right)$ to the essentially classical form of the Lagrangian (15), indicative of higher powers of the scalar curvature, could not be extracted reliably from small-volume measurements, which are subject to both finite-size effects and lattice artefacts.

In a follow-up investigation [77 the matrix elements (14) were extracted from measurements of a periodically identified two-slice system with $t_{\text {tot }}=2$. It established that in phase $C$ one can to good accuracy reconstruct from these data both the average volume profile $\left\langle n_{t}\right\rangle \propto \cos ^{3}(t /$ const $)$ and the covariance matrix

$$
C_{t t^{\prime}}=\left\langle\left(n_{t}-\left\langle n_{t}\right\rangle\right)\left(n_{t^{\prime}}-\left\langle n_{t^{\prime}}\right\rangle\right)\right\rangle
$$

previously measured in full CDT simulations 18 . The passing of this important consistency check raised the interesting question of whether the effective transfer matrix can be used as a new tool to learn more about the rest of the phase space of CDT quantum gravity. Trying to fit the measured matrix elements at the point $\left(\kappa_{0}, \Delta\right)=(5.0,0.4)$ inside phase $A$ to an effective Lagrangian similar to 15 produced evidence for a Lagrangian of the form

$$
L_{\mathrm{eff}}^{(A)}[n, m]=\mu\left(n^{\alpha}+m^{\alpha}\right)-\lambda(n+m),
$$

apparently without a kinetic term, and with a best fit yielding $\alpha \approx 0.5[77]$. However, one should keep in mind that phase $A$ most likely does not have any physical interpretation in terms of either gravity or (higher-dimensional) geometry, which means that an ansatz inspired by such an assumption may lead to misleading conclusions. Another finding of $[77]$ has a more straightforward interpretation: the kinetic term in the Lagrangian (15) vanishes gradually as one approaches the $A-C$ transition at fixed $\Delta$ from inside phase $C$ and is zero at the transition. This is consistent with the idea that inside phase $C$ the entropy of the microscopic configurations prevents the system from developing a conformal divergence, but that for increasing $\kappa_{0}$ the negative kinetic term of the conformal mode in the bare action will eventually become dominant [54,78].

While the $A-C$ transition is thus associated with a clear signal in the effective Lagrangian for the spatial volume, this does not seem to be true for the $B-C$ transition, although the latter $i s$ visible in the effective transfer matrix approach when looking at other order parameters (c.f. footnote 15).

Instead, monitoring the effective transfer matrix throughout phase $C$ as phase $B$ is approached has revealed the existence of a new phase transition inside phase $C$. This implies that the previous phase $C$ is now divided into the de Sitter phase

\footnotetext{
${ }^{18}$ Strictly speaking, this involved an extrapolation for the matrix elements with large arguments $n_{t}>700$, which appears well justified, see 77 for details.
} 


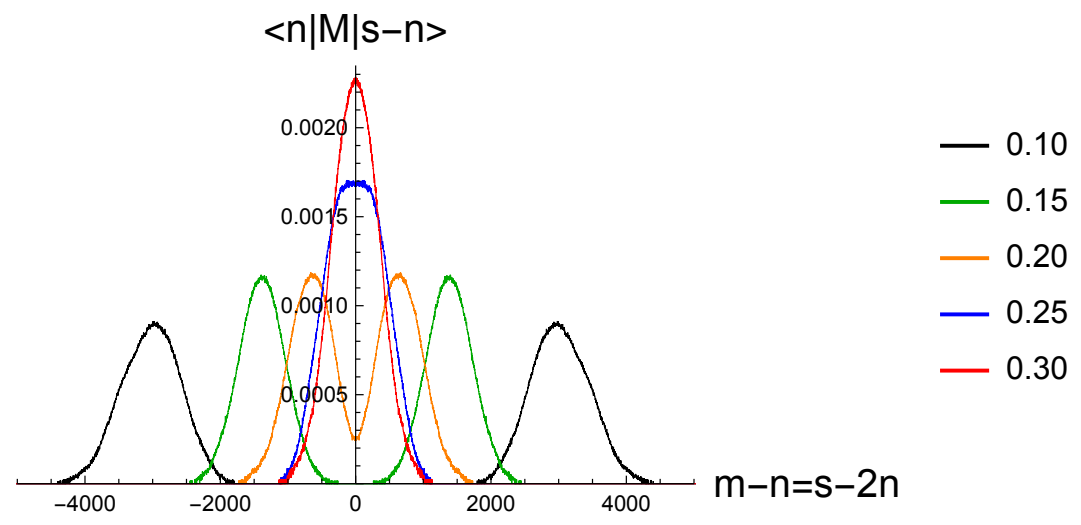

Figure 8: Measured matrix elements $\langle n|M| s-n\rangle$ for $s=30 k$, at fixed $\kappa_{0}=2.2$ and for five different $\Delta$-values. Since $s=m+n$ stays constant, these measurements illustrate the behaviour of the kinetic part of (18): the smaller $\Delta$, the more pronounced is the "bifurcation" into two Gaussians. The $C_{b}-C_{d S}$ phase transition (merger to a single Gaussian) appears to lie in the interval $\Delta \in[0.25,0.3]$. [Figure from [67].]

$C_{d S}$ and the bifurcation phase $C_{b}$, as indicated in the phase diagram of Fig. 5 . The name of the latter derives from a property of the effective transfer matrix, whose kinetic part $\exp \left[-(n-m)^{2} /(\Gamma(n+m))\right]$ in the bifurcation phase splits into a sum of two Gaussians with a relative shift, giving rise to matrix elements of the form

$$
\begin{aligned}
\langle n|M| m\rangle=[\exp (- & \left.\frac{1}{\Gamma} \frac{((n-m)-c[n+m])^{2}}{n+m}\right) \\
& \left.+\exp \left(-\frac{1}{\Gamma} \frac{((n-m)+c[n+m])^{2}}{n+m}\right)\right] \mathcal{V}[n+m],
\end{aligned}
$$

where $\mathcal{V}[n+m]$ is the exponential of the potential part of eq. (15) or some variant thereof [67, 77]. The function $c[n+m]$ in (18) goes to $c_{0}\left(n+m+s_{b}\right)$ for large spatial volumes $n+m \gg s_{b}$ and to zero for small volumes $n+m \ll s_{b}$. The two parameters $c_{0}$ and $s_{b}$ depend on the bare coupling constants $\kappa_{0}$ and $\Delta$. Coming from inside $C_{b}$, the $C_{b}$ - $C_{d S}$ transition is associated with the limits $c_{0} \rightarrow 0$ and $s_{b} \rightarrow \infty$, for which the two Gaussians merge into one, resulting in the previous form (14) for the matrix elements. Going the other way in phase $C_{b}$, by lowering $\Delta$ for fixed $\kappa_{0}$, one eventually enters phase $B$. In the process, the two Gaussians of (18) move further apart in a smooth way [67]. This is consistent with the overall geometry in phase $B$, where the universe is known to collapse to a single time slice. Expanding the ansatz (18) for small $c_{0}$ and large $s_{b}$ and interpreting the result as 
an effective Lagrangian like (15), one finds for sufficiently large spatial volumes an "effective" kinetic term with a negative sign, which the authors of 67, 79 suggest to interpret as a scale-dependent effective signature change of the metric. Since the total volume is just one of an infinity of modes of the spatial metric (in the continuum), this is a rather far-reaching conjecture. It may be worth re-examining once the physics of the bifurcation phase is better understood.

The location of the new phase transition was first estimated from the vanishing of the parameter $c[s] \equiv c[m+n]$ appearing in the exponentials on the right-hand side of eq. (18), in a two-slice system with volumes of up to $s=60 k$ [67]. The existence of the new $C_{b}-C_{d S}$ transition line was confirmed in simulations at fixed volumes $N_{41}=80 k$ and $160 k$ for a standard system of CDT configurations with $t_{\text {tot }}=80$ [71]. Its authors studied the behaviour of three order parameters near the transition, $\operatorname{conj}(\Delta)$ of eq. (11), and two new quantities introduced in [67], which both refer to a particular spatial slice at time $t_{0} 19$ They are

$$
\begin{aligned}
& O P_{1}:=\left|\bar{R}\left(t_{0}\right)-\bar{R}\left(t_{0}+1\right)\right|, \\
& O P_{2}:=\mid \max \left[O\left(v\left(t_{0}\right)\right)\right]-\max \left[O\left(v\left(t_{0}+1\right)\right] \mid,\right.
\end{aligned}
$$

where up to an irrelevant constant $\bar{R}(t)=2 \pi N_{0}(t) / N_{3}(t)$ is the average scalar curvature of the spatial slice at integer time $t$, using the deficit angle prescription of Regge calculus [35], and $\max [O(v(t))]$ denotes the maximal coordination number (the number of four-simplices meeting at $v$ ) of any vertex $v$ contained in the slice $t$. The choice of the new order parameters is motivated by a distinct "modulation" of several geometric properties of the CDT configurations in phase $C_{b}$, with characteristic time period $\Delta t=2$. For example, the maximal coordination number of any vertex in a given spatial slice oscillates strongly between alternating slices (Fig. 9p.

Varying $\Delta$ at fixed $\kappa_{0}=2.2$ and for $N_{41}=160 k$, its (pseudo-)critical value at the new phase transition was estimated as $\Delta^{\text {crit }}=0.35 \pm 0.01$ from the peaks of the susceptibilities of the parameters (19) and (20) [71]. A preliminary investigation into the order of the transition in the same work did not yield conclusive results, but a subsequent, comprehensive analysis of several indicators established strong evidence that the $C_{b}-C_{d S}$ transition is of second or higher order [80]. The indicators measured were the dependence of the pseudo-critical point $\Delta^{\text {crit }}$ on the lattice volume (the shift exponent used earlier for the other transitions [15, 16]), the peak separation in the histogram of the normalized order parameter $\overline{O P}_{2} / N_{41}$ as a function of the volume, and the frequency of parameter jumps of $O P_{2}$ as a function of Monte Carlo time, normalized by the autocorrelation time. Notable for the simulations near the transition is a severe critical slowing-down, associated with very long autocorrelation times, as documented in [71, 80].

\footnotetext{
${ }^{19}$ the slice closest to the maximum of the volume profile $n_{t}$ of a given configuration, or some variant involving the slice with the vertex of highest order, see [67,71,80 for details
} 


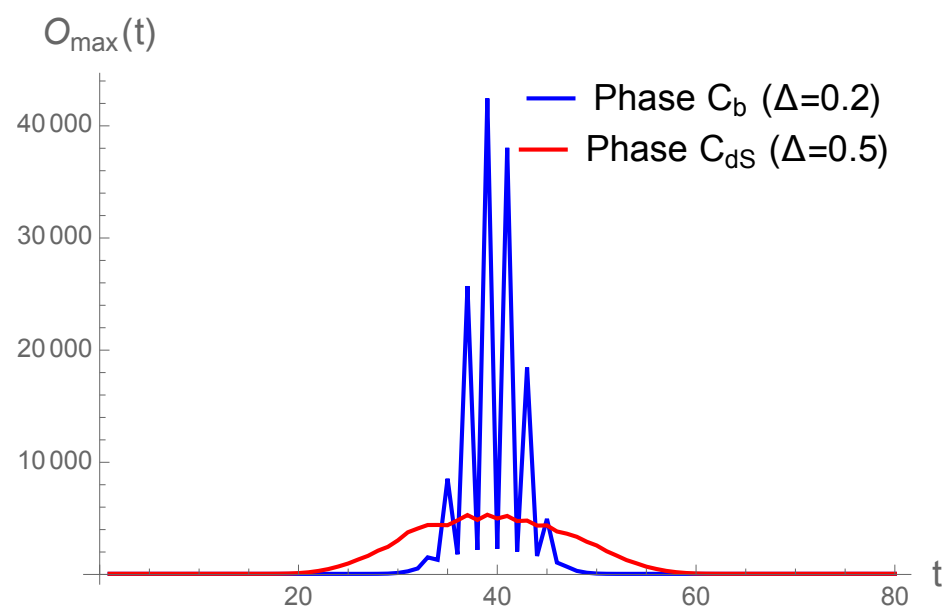

Figure 9: In the bifurcation phase $C_{b}(\Delta=0.2)$, the maximal coordination number $O_{\max }(t):=\max [O(v(t))]$ jumps to a very large value on every second spatial slice, compared with the situation in the de Sitter phase $C_{d S}(\Delta=0.5)$. Measurements taken for $t_{t o t}=80, N_{41}=160 k, \kappa_{0}=2.2$, and averaged over many configurations. [Figure from [80].]

\subsection{The bifurcation phase}

Before the discovery of the bifurcation phase, much, though by far not all, of the analysis of CDT geometry in the "well-behaved" $C$-phase was done at the point $\left(\kappa_{0}, \Delta\right)=(2.2,0.6)$, thought of as a generic point well inside phase $C$. This analysis, which includes the classic results of the de Sitter volume profile, with corresponding quantum fluctuations, and the dynamical dimensional reduction on short scales, remains valid inside the de Sitter phase $C_{d S}$, where this point is located. On the other hand, scaling behaviour compatible with a four-dimensional universe on large scales and a de Sitter-like behaviour were also found in at least part of what is now identified as phase $C_{b}$ (this is exactly why the $C_{b}-C_{d S}$ transition remained undetected for a long time). For the time being the question remains open of whether and to what extent - in addition to the de Sitter phase - also the bifurcation phase is interesting from a quantum gravity point of view, and has a good classical limit. More details need to be known on how the two phases differ in their geometric properties, which will in turn help us understand the nature of the new phase transition.

There is a particular large-scale property that has already been identified as characteristic for the bifurcation phase, and has motivated the introduction of the order parameters (19) and (20). Both measure a difference in geometry of adjacent spatial slices, which causes the effective bifurcation behaviour expressed by the functional form of the matrix elements (18). As first noted in [67], inside 

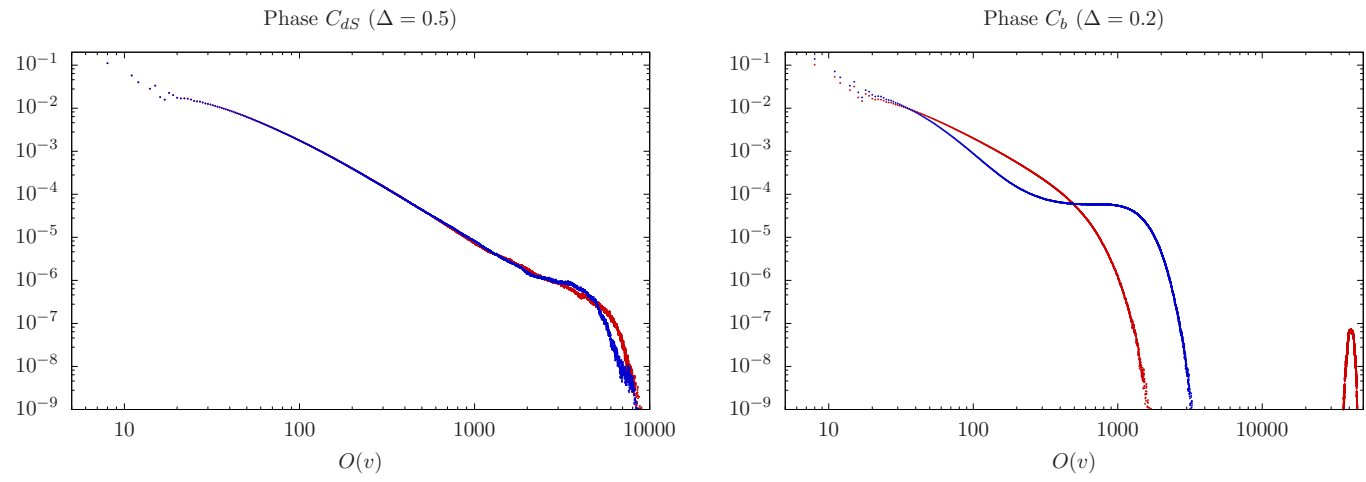

Figure 10: Histograms of the coordination number $O(v)$ in the spatial slice containing the vertex with maximal coordination number (red curves) and in a neighbouring spatial slice (blue curves). In the de Sitter phase $(\Delta=0.5$, left) there is hardly any difference. In the bifurcation phase $(\Delta=0.2$, right), the singular vertices form a peak in $O(v)$ isolated from the rest of the distribution, which is not present in slices without singular vertices. Measurements taken for $t_{t o t}=80, N_{41}=160 k$ and $\kappa_{0}=2.2$. [Figures courtesy of A. Görlich.]

phase $C_{b}$ each second slice appears to have a single "singular" vertex ${ }^{20}$ with an exceptionally high coordination number, both in a three- and a four-dimensional sense, reflected by the parameters (19) and (20) respectively, and illustrated by Fig. 9 above. Coming from the de Sitter phase $C_{d S}$ and moving into $C_{b}$ by decreasing $\Delta$, within a spatial slice that contains a singular vertex a gap opens between the coordination number $O(v)$ of this vertex and the coordination number of the vertex with the second-largest $O(v)$. The histograms of Fig. 10 illustrate this phenomenon, which is absent in the de Sitter phase. Well inside the bifurcation phase, in a slice containing such an exceptional vertex, its coordination number is a couple of orders of magnitude larger than the average coordination number of the slice.

The relation between maximal vertex order and spatial volume was made quantitative in [68, which for a system with $t_{t o t}=2, \kappa_{0}=2.2, \Delta=0$ and $N_{4}=$ $10 k$ found an approximately linear relation between the expectation value of the highest vertex order, located in slice $t_{1}$, say, and the volume difference $n_{t_{2}}-n_{t_{1}}$ with the neighbouring slice $t_{2}$. In other words, a high coordination number of a singular vertex in one slice is associated with a large volume in an adjacent slice (which does not have a singular vertex).

The overall picture that emerges in the bifurcation phase for CDT configu-

20 "Exceptional" instead of "singular" would be more appropriate, since these vertices are perfectly regular from the point of view of finite, piecewise flat geometries. We will nevertheless use this notion, first coined in the context of Euclidean Dynamical Triangulations 81. 

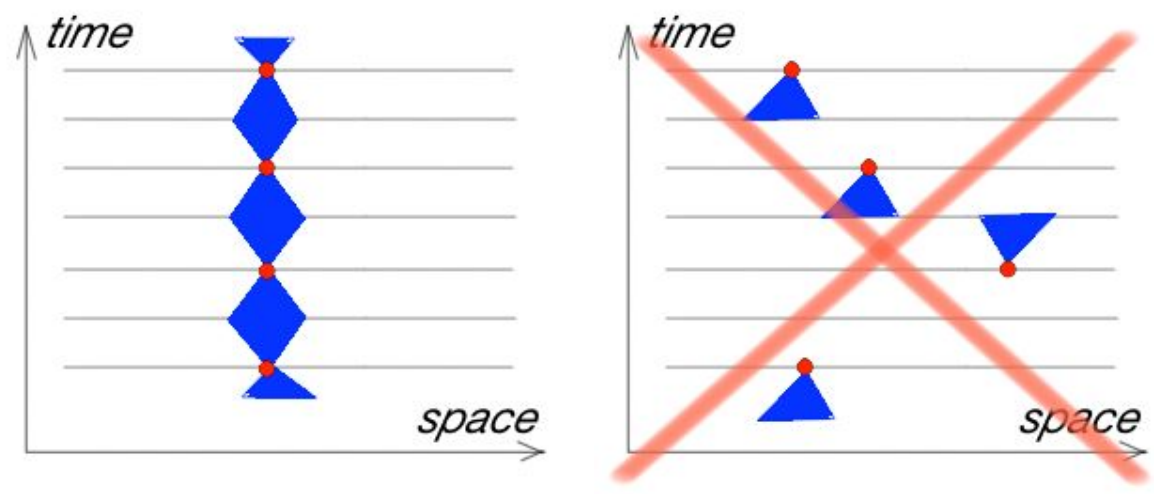

Figure 11: Schematic illustration of the spacetime geometry inside the bifurcation phase, where the singular vertices present on every second spatial slice align themselves into a chain of diamond-like regions, as shown in the figure on the left. [Figures from [67].]

rations with large time extension $t_{t o t}$ is that the singular vertices on alternating spatial slices are associated with a four-dimensional substructure of the triangulation, which consists of a chain along the time direction of roughly diamond-shaped regions. The tips of each "diamond" are a pair of singular vertices a distance $\Delta t=2$ apart, say, at times $t$ and $t+2$, and its body by definition consists of all four-simplices in the interval $[t, t+2]$ that contain either of the two singular vertices. They form a diamond because the two sets of four-simplices, lying in either $[t, t+1]$ or $[t+1, t+2]$, turn out to have a large overlap at the intermediate time $t+1$, in the form of shared (sub-)simplices. The situation is illustrated schematically by Fig. 11. The entire chain forms a substructure imbedded in the rest of the triangulation, and contains a large, finite fraction of the total four-volume.

As already remarked in 67], the singular vertices and their associated substructures in $C_{b}$ break the spatial homogeneity and isotropy that appear to be present (in a statistical sense, and on sufficiently large scales) in the de Sitter phase and presumably are related to the fact that the large-scale properties of the dynamically generated quantum universe in $C_{d S}$ are very well described by a minisuperspace model with built-in homogeneity and isotropy [8, 9, 11]. For this reason, the new $C_{b}-C_{d S}$ phase transition has been associated tentatively with the breaking of this symmetry $67,68,80$.

\subsection{CDT on a spatial torus}

As already mentioned in Sec. 5, CDT configurations satisfy a lattice version of global hyperbolicity, which entails a fixed topology ${ }^{(3)} \Sigma$ for the three-dimensional spatial slices at fixed time $t$. All results discussed until now have used a spherical 
topology, ${ }^{(3)} \Sigma=S^{3}$, and - for the convenience of not having to deal with boundaries in the simulations - a compactified time direction, tantamount to a global topology $S^{1} \times S^{3}$. Since this amounts to a specific choice that is made a priori, the question arises of whether and to what extent a different choice of spatial topology will affect any of the key findings of CDT quantum gravity. On the one hand, one would not expect the global topology to affect local physics, at least not for sufficiently large pieces of spacetime. On the other hand the properties of quantum gravity are studied with the help of observables that themselves are often very nonlocal. ${ }^{21}$ Given the limited range of scales accessible in computer simulations, this means that disentangling local and global features will in general be nontrivial.

Several results for CDT with a spatial three-torus, ${ }^{(3)} \Sigma=T^{3}$, are now available [82 84. The analysis proceeds largely along the lines of the spherical case and so far has included investigations of the volume profile, the associated effective transfer matrix and effective action, and mapping out the phase diagram. As will be described in more detail below, the dynamics of the global scale factor (the three-volume) is sensitive to the global topology, while the number, location and broad characteristics of the phases are not, in line with the expectation articulated in the previous paragraph. Unlike what happens for spherical spatial slices, where the chosen topology $S^{1} \times S^{3}$ appears to be driven dynamically to that of a four-sphere $S^{4}$, for toroidal spatial slices the chosen topology $S^{1} \times T^{3}$ is unchanged, at least in the phase(s) with macroscopic semiclassical geometry.

One reason why the three-torus is not the first choice in simulations is that the minimal size of a simplicial three-manifold with this topology (in terms of the number of equilateral tetrahedra it contains) is much bigger than that of a three-sphere, namely, 90 as opposed to $5[82$. This is bound to reduce the window between lattice artefacts and finite-size effects where reliable measurements can be made and, generally speaking, will drive up the lattice volumes at which specific phenomena are observed, compared to the spherical case. This effect has been confirmed in the actual simulations.

The simulations require configurations with spacetime topology $S^{1} \times T^{3}$, consisting of $t_{t o t}$ individual sandwich geometries $I \times T^{3}$. Reference [82] provides an explicit construction of an initial configuration of this kind, which is needed as a starting point for the Monte Carlo simulations. It is obtained by gluing together regular hypercubes subdivided into four-simplices. Each sandwich geometry is a layer of thickness $\Delta t=1$ of these cubes, assembled in a specific way to make sure that the triangulated three-geometries shared by adjacent layers match. Each sandwich contains a total of 384 four-simplices of type $(3,2)$ and 640 of type

\footnotetext{
${ }^{21}$ Note that "nonlocal" here is not meant in the sense of causality-violating, faster-than-light propagation of information, to the extent that this is an operationally well-defined concept at Planckian distance scales in the first place.
} 

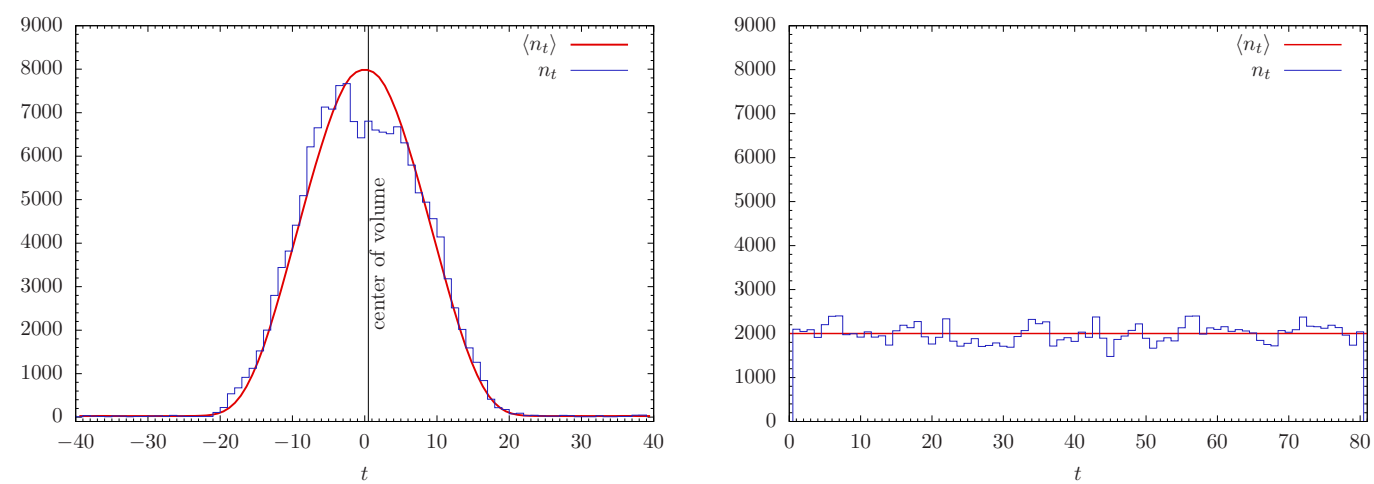

Figure 12: Snapshot of a typical volume configuration $n_{t}$ (blue) and the expectation value $\left\langle n_{t}\right\rangle$ (red), both for spherical slices (left) and toroidal ones (right). All measurements taken at $\left(\kappa_{0}, \Delta\right)=(2.2,0.6)$, and for $N_{41}=160 k$ and $t_{\text {tot }}=80$. [Figures from [83].]

$(4,1)$, and its spatial boundaries consist of 320 equilateral tetrahedra each. Using the same set of ergodic Monte Carlo moves as for $S^{1} \times S^{3}$, this initial spacetime configuration is grown until a target volume $\bar{N}_{41}$ is reached at which the simulations are performed in the usual manner.

First simulation results of the toroidal system at couplings $\left(\kappa_{0}, \Delta\right)=(2.2,0.6)$, $t_{t o t}=80$ and with a quadratic volume fixing (10) were reported in [82. Unlike for the spherical system, for which this point is associated with a nontrivial expectation value $\left\langle n_{t}\right\rangle \propto \cos ^{3}(t /$ const $)$ for the volume profile, the corresponding quantity for the spatial torus universe appears to be time-independent, $\left\langle n_{t}\right\rangle \propto$ const. Fig. 12 illustrates both typical configurations $n_{t}$ for the two systems as well as their mean values $\left\langle n_{t}\right\rangle$. In the spherical case, the effective time extension of the de Sitter universe (the time interval where the spatial volume differs appreciably from its kinematically allowed minimum $n=5$ ) scales $\propto N_{4}^{1 / 4}$ as a function of the total four-volume $N_{4}$, while for the spatial volumes one finds $n \propto N_{4}^{3 / 4}$ [8, 11, 17]. The situation for the torus case is different: the four-volume is on average evenly distributed over the available layers, such that $n \propto N_{4} / t_{t o t}$.

For small perturbations $\Delta n_{t}=n_{t}-\left\langle n_{t}\right\rangle$ around the constant average threevolume, one can again try to determine an associated effective action from measuring the volume-volume covariance matrix (16) and taking its inverse. The elements of neither the covariance matrices nor their inverses show any systematic time dependence. Taking time averages of the diagonal and sub- and superdiagonal elements of the inverse covariance matrix, and studying their dependence on the slice volume (by varying the four-volume in the range $N_{41} \in[80 k, 240 k]$ and the time extension in the range $\left.t_{t o t} \in[10,200]\right)$ resulted in an effective action of 
the form

$$
S_{\mathrm{eff}}=\sum_{t}\left(\frac{1}{\Gamma} \frac{\left(n_{t}-n_{t+1}\right)^{2}}{n_{t}+n_{t+1}}+\mu n_{t}^{-\gamma}+\lambda n_{t}\right),
$$

with $\Gamma \approx 26.3, \gamma=1.16 \pm 0.02$ and $\mu>0\left[82 .{ }^{22}\right.$ Comparing this with the effective Lagrangian (15) obtained for the spherical case, the kinetic term is identical, including the value of $\Gamma$ within measuring accuracy. However, the potential term proportional to $n^{1 / 3}$ is absent in the torus case. This latter finding is in agreement with the classical (Euclidean) minisuperspace action for FLRW metrics with flat toroidal slices in proper-time form,

$$
d s^{2}=d t^{2}+a^{2}(t)\left(d x^{2}+d y^{2}+d z^{2}\right)
$$

given by

$$
S_{e u}^{\mathrm{EH}} \propto \frac{1}{G_{\mathrm{N}}} \int d t\left(a \dot{a}^{2}-\frac{\Lambda}{3} a^{3}\right)
$$

obtained by inserting the ansatz (22) into the Euclidean counterpart of the continuum Einstein-Hilbert action (2). By contrast, the integrand of the corresponding minisuperspace action on spherical slices (of constant positive curvature) has an additional potential term linear in the scale factor $a(t)$, which re-expressed in terms of the three-volume corresponds to a term $\propto n^{1 / 3}$. It is remarkable that the nonperturbative CDT quantum theory with both spherical and toroidal spatial topology reproduces these detailed features of the potential terms of the corresponding classical minisuperspace cosmologies. The term with negative power $n^{-\gamma}$ in eq. (21) does not appear in the classical action and is interpreted as a genuine quantum correction. Such a term may in principle also be present in the spherical case, but is out of reach with current measurement precision. There is at this time no analytic argument for any particular value of the exponent $\gamma$.

These results were confirmed in a second, more detailed study, where the average volume profile was forced to be nonconstant, to allow for measurements at different slice volumes within the same Monte Carlo simulation [83. Instead of fixing the total volume, one (approximately) fixes the spatial volume at two times $t$ and $t^{\prime}$ to some target volumes $\bar{n}_{t}$ and $\bar{n}_{t^{\prime}}$ by adding a term

$$
S_{\text {fix }}=\epsilon\left[\left(n_{t}-\bar{n}_{t}\right)^{2}+\left(n_{t^{\prime}}-\bar{n}_{t^{\prime}}\right)^{2}\right], \quad \epsilon>0,
$$

to the bare action. The numerical study used $t_{t o t}=40$, and fixed the volumes at times $t=1$ and $t^{\prime}=21$ (Fig. 13). For this system, the inverse covariance matrix was again determined, confirming the functional form (21) of the effective

${ }^{22} \mathrm{~A}$ cosmological constant term is included here for generality; $\lambda$ does not play a role in the fixed-volume study of 82 , other than possibly as a Lagrange multiplier. It has been determined in the effective transfer matrix treatment of 83 as $\lambda \approx 3.5 \times 10^{-4}$. 


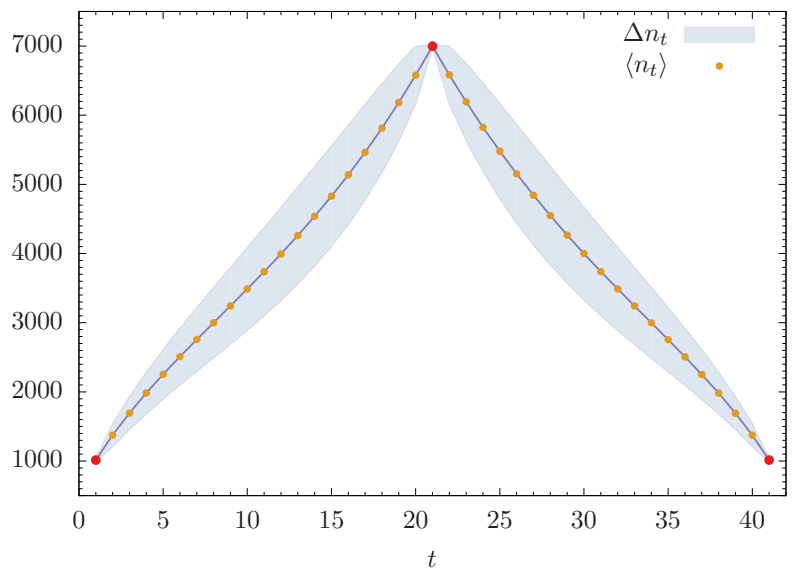

Figure 13: Volume profile $\left\langle n_{t}\right\rangle$ of CDT configurations with $t_{t o t}=40$ toroidal slices, with fixed spatial volumes $\bar{n}_{1}=1000$ and $\bar{n}_{21}=7000$. The shaded region indicates the size of the quantum fluctuations of $n_{t}$ around the mean. Measurements taken at $\left(\kappa_{0}, \Delta\right)=(2.2,0.6)$ and $\kappa_{4}=0.9225$. [Figure from [83].]

action, with $\Gamma=26.2 \pm 0.1$ and $\gamma \approx 1.5$, in reasonable agreement with the previous results [82]. The shape of the volume profile $\left\langle n_{t}\right\rangle$ of Fig. 13 can be matched qualitatively well by classical solutions derived for the continuum analogue of the action (21) 83.

The effective transfer matrix method introduced in Sec. 6.1 above has also been applied to CDT with spatial tori, yielding compatible results for the volume behaviour. The matrix elements $\langle n|M| m\rangle$ have been measured in Monte Carlo simulations of the system with $t_{t o t}=2$, at the phase space point $\left(\kappa_{0}, \Delta\right)=(2.2,0.6)$ and for various fixed values of the volume $N_{41}=m+n$. The results are well approximated by an effective Lagrangian of the form

$$
L_{\mathrm{eff}}[n, m]=\frac{1}{\Gamma} \frac{(n-m)^{2}}{(n+m)}+\mu\left(n^{-\gamma}+m^{-\gamma}\right)+\lambda(n+m) .
$$

Alternatively, the same Lagrangian with a potential term $\mu(n+m)^{-\gamma}$ instead of $\mu\left(n^{-\gamma}+m^{-\gamma}\right)$ is an equally good fit to the data. Following the strategy developed in [77], it has been checked to what extent the volume profile $\left\langle n_{t}\right\rangle$ and the amplitude of the fluctuations $\Delta n_{t}$ obtained for the time extension $t_{t o t}=40$ (Fig. 13) can be reconstructed from the matrix elements $\langle n|M| m\rangle$ of the effective transfer matrix alone [83]. It turns out that the volume profile can be reproduced well for a suitable choice of $\kappa_{4}$. The curve of the amplitude of the fluctuations looks qualitatively similar, but there is a noticeable, small discrepancy. This indicates that excitations other than the three-volume induce correlations beyond pairs of neighbouring slices that are not captured by the effective volume dynamics. 
Phase A

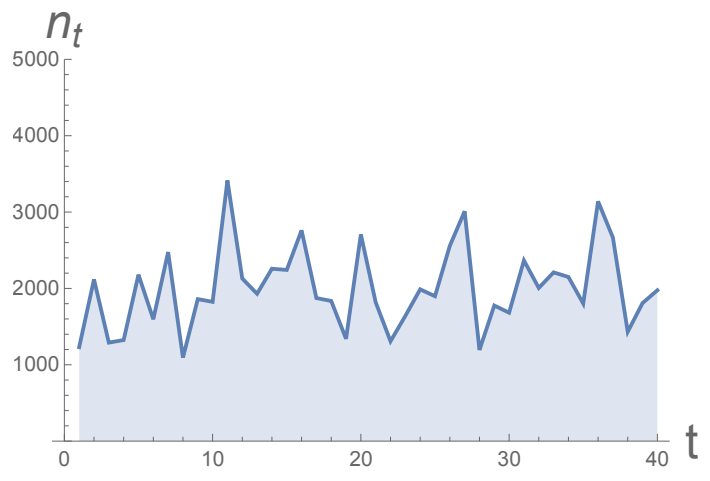

Phase B

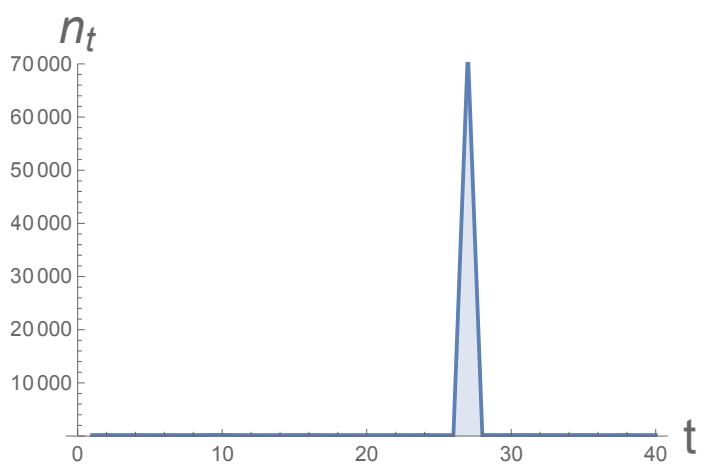

Figure 14: Sample volume profiles $n_{t}$ of CDT configurations with $t_{\text {tot }}=40$ toroidal slices and for $N_{41}=40 k$ display characteristics familiar from phases $A$ and $B$ of the spherical system. [Figures from [84].]

The phase structure of the toroidal system was investigated in [84], starting with a grid-like sampling of the $\left(\kappa_{0}, \Delta\right)$-space for fixed lattice volume $N_{41}=80 \mathrm{k}$ and $t_{t o t}=40$. For this purpose, one employs a set of order parameters,

$$
N_{0} / N_{4}, \quad N_{32} / N_{41}, \sum_{t \in\left[1, t_{t o t}\right]}\left(n_{t}-n_{t-1}\right)^{2}, \quad \max _{v \in T} O(v)
$$

which are variants of previously used parameters also applicable to constant volume profiles. Scanning the phase space to establish in which regions these parameters (or suitably rescaled versions) are large or small one finds an overall picture that is qualitatively very similar to that for spherical slices, with typical characteristics of the $A-, B$ - and $C$-phases. For example, as $\Delta$ is increased and $\kappa_{0}$ held fixed, $N_{0} / N_{4}$ increases and the last two order parameters in 26) decrease monotonically. One also observes volume profiles $n_{t}$ that are reminiscent of those of the unphysical phases $A$ and $B$ of the sphere case (Fig. 14). In the former, the entire universe collapses in time, with only a single spatial slice whose volume is nonvanishing, and the latter is associated with large, random volume differences between adjacent slices (in both cases subject to the kinematical minimal-volume constraint for the spatial tori).

It is perhaps unsurprising that neither the bifurcation phase nor an analogue of the $C_{b^{-}} C_{d S}$ phase transition are visible in these data; since the typical slice volumes are of the order of 2000, and the volume profile is approximately constant in phase $C$, there is likely not enough three-volume to allow singular vertices to develop. This has motivated a further study of the torus model with short time extension $t_{t o t}=4$, at total volume $N_{41}=160 k$ [84]. In this set-up, the characteristic structure of the bifurcation phase is visible, with vertices of very high 


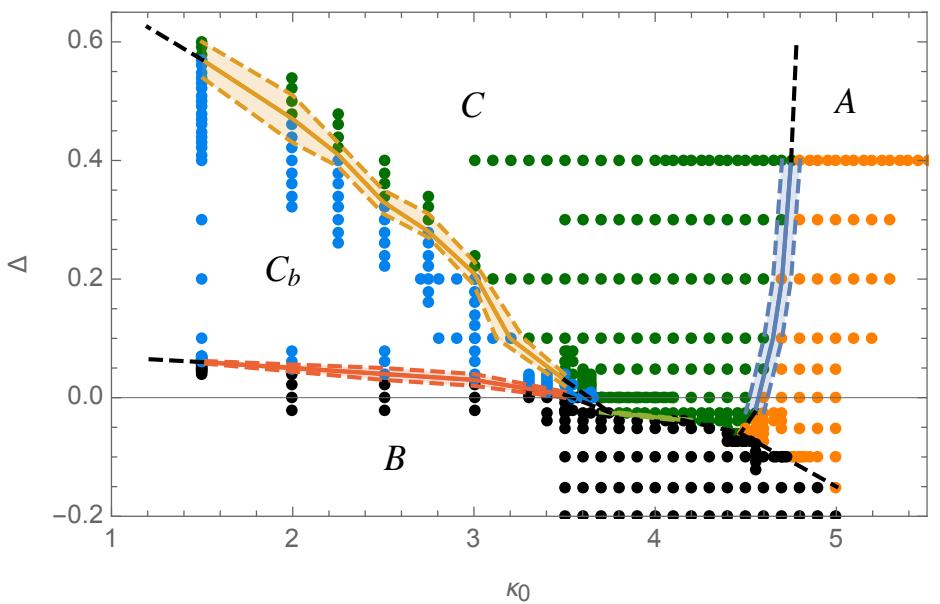

Figure 15: The phase diagram of CDT quantum gravity with $t_{\text {tot }}=4$ toroidal slices, from measurements at volume $N_{41}=160 k$. Phase transitions are marked by solid lines, with shaded areas of the same colour indicating error bars. Dots mark measurement points, with different colours associated to the four different phases. The phase denoted $C$ is the analogue of the de Sitter phase for spherical slices. Dashed black lines indicate extrapolations. [Figure from [84].]

coordination number $(\sim 60 k)$ forming on every second spatial slice. In addition, extensive measurements of both the order parameters (26) and their susceptibilities have been made for these configurations, achieving a high resolution of the location of the phase transitions. The resulting phase diagram is shown in Fig. 15. Its structure is clearly very similar to that for CDT quantum gravity for spherical slices (Fig. 5), despite the fact that one can still expect significant shifts in the location of the transition lines, due to strong finite-size effects for the torus measurements. One interesting difference with the spherical case is that the critical slowing-down observed near the bottom right-hand corner of the phase diagram, where various transition lines meet, is much less pronounced. This will provide a good starting point for measurements of the order of the phase transitions, which in general are anticipated to be computationally expensive 84 .

\subsection{Renormalization group flow in CDT}

In the absence of an a priori defined length scale, it is not a given that renormalization group arguments can be formulated consistently in a backgroundindependent theory of quantum gravity, where any notion of scale will typically have to be generated dynamically. However, a proof of principle has been given in 85] that these difficulties can be overcome in CDT quantum gravity. One follows a strategy similar to what is done in the lattice formulation of quantum fields 
on a fixed flat background, say, of scalar fields with a $\lambda \phi^{4}$-interaction (see, for example, [86]). The key idea is to identify paths of constant physics in the space of bare coupling constants, follow them in the direction of decreasing lattice spacing or, equivalently, increasing correlation length, and investigate whether they approach second-order transition points or lines where a continuum limit can be taken. In the case of gravity, it would be highly interesting to verify the presence of UV fixed points of the renormalization group, as predicted by asymptotic safety, and to investigate the physical properties of the system in the approach to such a fixed point.

Clearly, constant physics must be defined in terms of observables, which are much easier to identify in scalar field theory than in quantum gravity. A limiting factor in the renormalization group investigations at this stage is the scarcity of quantum-gravitational observables, as well as the unclear phenomenological status of some of them, for example, a quantity like the spectral dimension. The analysis of [85] is based on observables with a clear-cut interpretation in terms of macroscopic physics, at least inside phase $C$, where the macroscopic shape of spacetime can be matched to that of a de Sitter universe ${ }^{23}$ They are the total four-volume of spacetime, the three-volume of the spatial universe at constant proper time, and the quantum fluctuations of the three-volume around the mean of the latter.

We currently do not have a good gravitational counterpart of a correlation length, which is the observable used to set the scale in standard lattice field theory, in the sense of providing an identification of lattice units with dimensionful physical units. Instead, [85] uses the quantum fluctuations of the spatial volume as a physical yardstick, and identifies lines of constant physics as those with a constant ratio of the average size of these quantum fluctuations and the average size of the spatial universe ${ }^{24}$ Fig. 16 illustrates how flow lines of constant physics are extracted in phase $C$. One further assumption underlying the analysis of [85] is that the ratio of the proper length of a space-like and a time-like lattice unit does not depend on the values of the bare coupling constants. This is arguably the most straightforward assumption, but certainly not the only one possible (see also [88 for a related discussion). It illustrates the subtleties of studying renormalization group flows in background-independent quantum gravity: in a situation where scales are generated dynamically, their possible dependence on the bare coupling constants must be analyzed with care, since it may affect the definition of what is meant by constant physics.

Note that the study 85 predated the discovery of the $C_{b^{-}} C_{d S}$ phase transi-

\footnotetext{
${ }^{23}$ Note that 85 interprets the measurements in the more general framework of HořavaLifshitz gravity, allowing also for a "deformed" de Sitter universe.

${ }^{24}$ Ref. 87. suggests the use of additional, alternative yardsticks, which one would like to extract from the behaviour of the spectral dimension, see Sec. 7.1 below for further comments.
} 

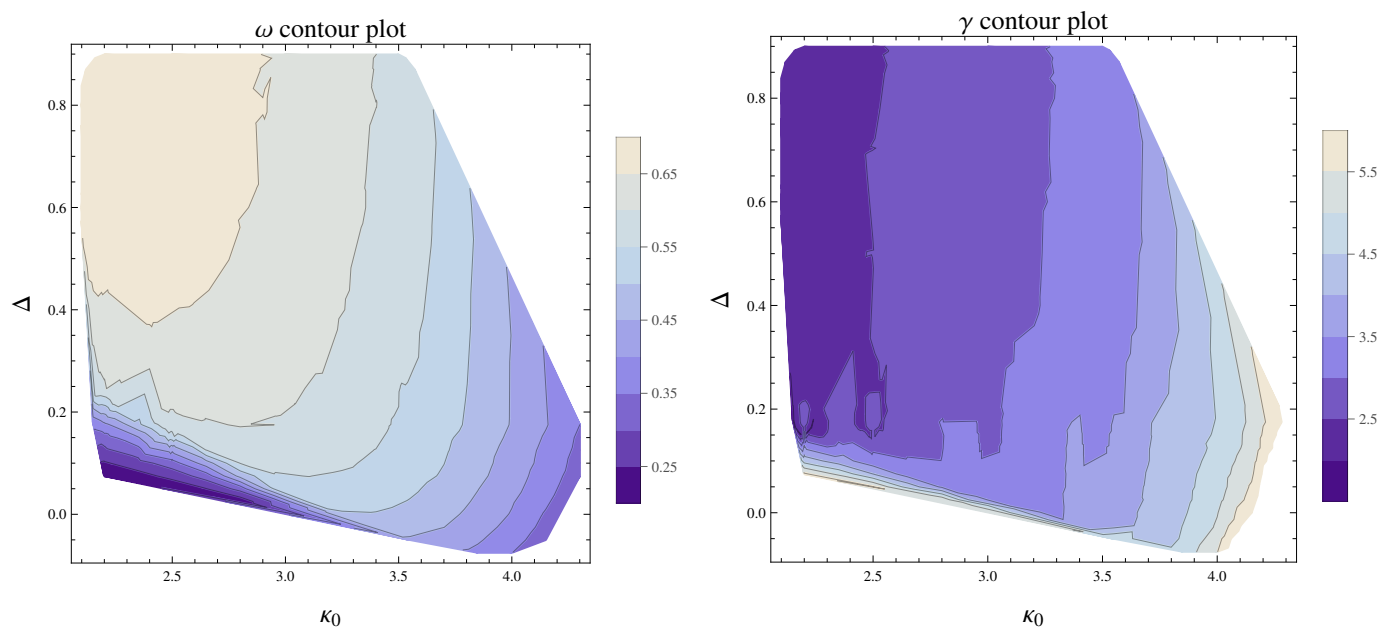

Figure 16: Lines of constant physics in the $\kappa_{0}-\Delta$ plane according to the renormalization group analysis of [85] are given by lines of constant $\omega$ (left) along which $\gamma$ (right) increases $\propto N_{4}^{1 / 4}$ in a scaling limit $N_{4} \rightarrow \infty$. The parameters $\omega$ and $\gamma$ are extracted from the average volume profile and its fluctuation spectrum.

tion, and therefore focused on the second-order $B-C_{b}$ transition. Its analysis did not yield evidence of flow lines running into this transition line. However, this result should not be taken as conclusive, since a critical slowing-down severely hampered the taking of data near the transition line and made it very computationally intensive. Besides improving computational algorithms, the scope for taking this research to the next level is clearly very rich, including other transition lines, alternative global set-ups (torus topology, transfer matrix setting), alternative scaling assumptions and - most importantly - more observables, together with a careful analysis of how they can yield reliable physical yardsticks. The larger value of the work of [85], which remains the only explicit nonperturbative renormalization group analysis in CDT quantum gravity, with an actual computation of renormalization group trajectories, is to provide a blueprint for a methodology that may well be relevant and applicable beyond this particular approach.

\section{Quantum-geometric observables}

As we have been emphasizing throughout this review, identifying and measuring observables is key to understanding the physical content of CDT quantum gravity and the existence and nature of its scaling limits in particular. Since the previous major review of the subject in [17], the already known observables have been 
studied in greater detail and generality and new ones have emerged. This section summarizes the most important new developments.

\subsection{Spectral observables}

Spectral observables refer to properties of the Laplace-Beltrami operator in the piecewise flat context of CDT, either on the full spacetime or on spatial slices of constant time. The most studied observable in this class is the spectral dimension $D_{S}$, whose original measurement in CDT quantum gravity in 10,11 exhibited the surprising phenomenon of dynamical dimensional reduction, namely, the smooth decrease of the spectral dimension from its classical value of 4 on large scales to a smaller value compatible with 2 (within error bars) as the Planck scale is approached. As has been described in detail elsewhere [10, 17], this result was established by studying a diffusion process on the ensemble of CDT geometries, more precisely, by measuring the expectation value of the average return probability $P_{g}(\sigma)$ of a random walker on a spacetime geometry $g$,

$$
P_{g}(\sigma)=\frac{1}{V_{g}} \int d^{4} \xi \sqrt{\operatorname{det} g(\xi)} K_{g}(\xi, \xi ; \sigma),
$$

for a suitable range of diffusion times $\sigma$. In eq. (27), $K_{g}\left(\xi, \xi_{0} ; \sigma\right)$ denotes the probability density of diffusion from a point $\xi_{0}$ to a point $\xi$ in diffusion time $\sigma$, and $V_{g}=\int d^{4} \xi \sqrt{\operatorname{det} g(\xi)}$ is the spacetime volume. The function $P_{g}(\sigma)$ is also called the heat trace or heat kernel trace. For a smooth, $d$-dimensional Riemannian geometry $g$, the (nonnegative) eigenvalues $\lambda_{i}$ of the Laplace-Beltrami operator $\Delta_{g}$ acting on scalar functions are related to the return probability by

$$
P_{g}(\sigma)=\frac{1}{V_{g}} \sum_{i} \mathrm{e}^{-\lambda_{i} \sigma},
$$

where degenerate eigenvalues appear separately in the sum and for simplicity we have assumed that the spectrum is discrete. At the same time, in the limit $\sigma \rightarrow 0$ there is an asymptotic expansion of $P_{g}(\sigma)$ of the form

$$
P_{g}(\sigma) \cong \frac{1}{(4 \pi \sigma)^{d / 2} V_{g}} \sum_{n=0}^{\infty} a_{n} \sigma^{n},
$$

where $a_{0}=V_{g}$ and the remaining $a_{n}$ are integrated curvature invariants of order $n$ [89]. We can extract the topological dimension $d$ of the Riemannian manifold by evaluating the logarithmic derivative of 29$)$ in the limit $\sigma \rightarrow 0$. More generally, we can define the spectral dimension function

$$
D_{S}(\sigma):=-2 \frac{d \log P_{g}(\sigma)}{d \log \sigma}
$$


for arbitrary diffusion times $\sigma \geq 0$. One easily computes that on an infinite flat space, eq. (30) evaluates to the topological dimension $D_{S}(\sigma)=d$, independent of $\sigma$. For general Riemannian spaces with nonvanishing curvature, it is clear from the expansion 29 that the spectral dimension $D_{S}(\sigma)$ away from $\sigma=0$ will in general differ from $d$, such that

$$
D_{S}(\sigma)=d+\text { correction terms, }
$$

where the "correction terms" can have either sign, depending on the values of the curvature invariants. The form of eq. 28) illustrates that for small $\sigma$ the function $D_{S}(\sigma)$ probes fine-grained geometric properties of the Riemannian space that are associated with the entire spectrum of the Laplace-Beltrami operator. By contrast, for large $\sigma$ only the low-lying part of the spectrum gives a significant contribution, which characterizes the large-scale geometry and topology of the space in question. In particular, on a compact space the smallest nonvanishing eigenvalue of $\Delta_{g}$ determines the rate of the exponential fall-off of the function $D_{S}(\sigma)$ for large $\sigma$. The linear scale associated with a given $\sigma$ is $\sqrt{\sigma}$, which is the typical linear distance travelled by a random walker away from its starting point after a diffusion time $\sigma$.

The relevance of this construction for quantum gravity and other applications of random geometry is the fact that Laplace-type operators and diffusion processes can be formulated in a much more general context than that of smooth Riemannian manifolds, including for example fractals and graphs. The aim of the original treatment in CDT quantum gravity [10,11] was to determine an effective spectral dimension of quantum spacetime at short distances from measuring the expectation value $\langle P(\sigma)\rangle$ of the return probability and then using (30), in other words, a quantum analogue of the limiting prescription $\sigma \rightarrow 0$ of $D_{S}(\sigma)$, which classically yielded the topological dimension $d$.

Since it is generally not the case that a nonperturbative superposition of geometries assembled from $d$-dimensional building blocks will give rise to an effectively $d$-dimensional geometry on any scale beyond the cut-off, the result that the short-scale spectral dimension measured in CDT is asymptotically compatible with 4 is highly nontrivial. On top of this came the surprising finding that at distances larger than the cut-off scale (and its associated lattice artefacts) but below the region where $D_{S}(\sigma)$ behaves classically, the spectral dimension undergoes a significant reduction, as already mentioned above. A natural interpretation of this phenomenon is in terms of quantum corrections to the spectral dimension in a Planckian, genuinely nonperturbative regime ${ }^{25}$ Note that in the $\sigma$-interval where the spectral dimension could be measured and its thermodynamic limit be

\footnotetext{
${ }^{25}$ Note that the Planck scale is not invoked in an ad hoc manner in CDT, but can be extracted from the measured spectrum of the quantum fluctuations of the spatial volume aka the "Friedmann factor".
} 


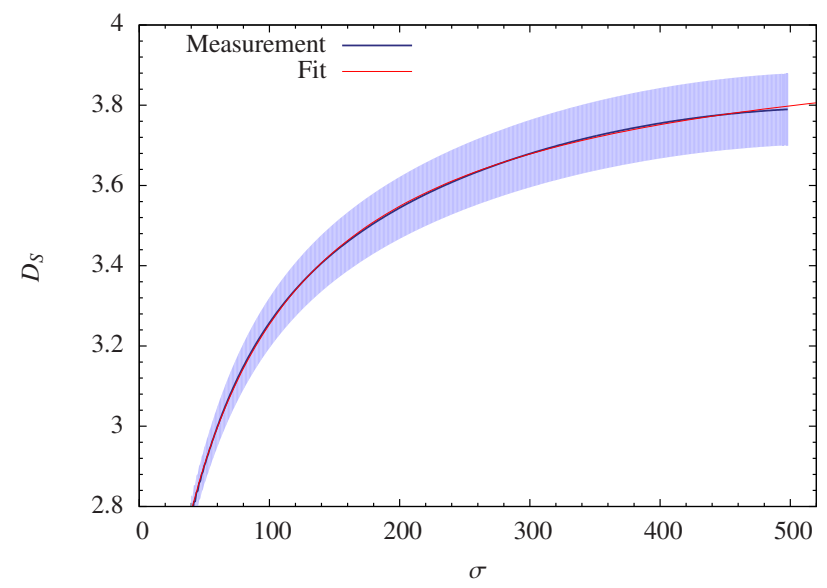

Figure 17: The spectral dimension $D_{S}$ of spacetime as a function of the diffusion time $\sigma$, measured at $\left(\kappa_{0}, \Delta\right)=(2.2,0.6)$, for $t_{\text {tot }}=80$ and a spacetime volume $N_{4}=181 k$. The averaged measurements lie along the central curve, together with a superimposed best fit $D_{S}(\sigma)=4.02-119 /(54+\sigma)$. The two outer curves represent error bars.

extracted with good confidence, $D_{S}(\sigma)$ takes on values $\lesssim 3.8$ (see Fig. 17) and therefore cannot be regarded as having reached its classical regime. Nevertheless, the existence of a classical regime and the scale dependence of $D_{S}(\sigma)$ were established robustly with the help of heuristic three-parameter fits in the range of $\sigma$ where measurements are reliable.

Since these early results, determining the short-scale spectral dimension has taken on a larger significance in nonperturbative quantum gravity, simply because it is a rare instance of a number that can be computed or measured in a large variety of candidate theories or, more generally, in nonclassical models of spacetime. This fact, and multiple indications - beyond CDT - that there may be a "true" or "correct" value $D_{S}(\sigma \rightarrow 0)=2$ (see, for example, [12]), make this reminiscent of computations of black hole entropy, whose value by contrast is a prediction of semi-classical gravity. On the one hand, the fact that "we finally can compute numbers" in quantum gravity, even if their phenomenological implications are unclear, should certainly be welcomed. On the other hand, some degree of caution is advised, considering that various derivations are performed in (sometimes highly) incomplete formulations of quantum gravity, rely heavily on additional ad hoc assumptions, employ semi-classical arguments of doubtful applicability, and/or are of a purely kinematical nature. In addition, some coincidences in the value of $D_{S}(\sigma \rightarrow 0)$ may be purely accidental, or are seen to occur only in particular (topological) dimensions (see [90] for a related discussion).

Recent developments regarding spectral observables in CDT include measure- 
ments of the spectral dimension away from the "canonical" phase space point $\left(\kappa_{0}, \Delta\right)=(2.2,0.6)$, in phases $C_{d S}$ and $A[91,92]$, the suggestion to investigate the function $D_{S}(\sigma)$ on all scales, generalising a corresponding study of CDT in three dimensions [90], and a comprehensive analysis of the spectral properties of three-dimensional spatial slices in four-dimensional CDT quantum gravity [50].

Ref. 91] corroborates the original result $D_{S} \approx 2$ for the short-distance spectral dimension at $\left(\kappa_{0}, \Delta\right)=(2.2,0.6)$, finding $D_{S}(\sigma \rightarrow 0)=1.97 \pm 0.27$ in simulations at roughly twice the previous volume, $N_{4}=367 k$, with time extension $t_{\text {tot }}=80$, and otherwise following the procedure outlined in [10]. The authors repeat the same measurement at three other points in phase $C_{d S}$ that are closer to the first-order $A-C_{d S}$ transition line, $\left(\kappa_{0}, \Delta\right)=(3.6,0.6),(4.4,0.6)$ and $(4.4,2.0)$, and for fixed $N_{41}$ of up to $300 k$. Moving closer to this line, the curves for $D_{S}(\sigma)$ flatten considerably in the $\sigma$-range considered $(\sigma \leq 500)$, making the fitting more challenging. Somewhat surprisingly, one finds $D_{S}(0) \approx 1.5$ at these points, within error bars, which seems at odds with the assumed universal character of this observable. On the other hand, one would expect short-distance properties to be closer to their universal values in the vicinity of a higher-order transition, which the new phase space points examined in [91 are not. Another possible explanation for the discrepancy is that the systematic errors are underestimated and that the fitting functions used are less appropriate in this region of phase space. Some evidence for this is the fact that the fitted curves for the spectral dimension functions corresponding to different phase space points do not match particularly well, even after a best rescaling of $\sigma$ (Fig. 3 in [91]). - In addition to measurements in the de Sitter phase, 91 also determined the spectral dimension of CDT at the point $\left(\kappa_{0}, \Delta\right)=(8.0,0.6)$ deep inside phase $A$, with strong indications of "branched-polymer" behaviour $D_{S} \approx 4 / 3$, independent of $\sigma$ in the range $\sigma \in$ [60, 500], see Fig. 18 .

A related line of argument is pursued in [92], where in addition to the phase space points $\left(\kappa_{0}, \Delta\right)=(2.2,0.6),(3.6,0.6)$ and $(4.4,0.6)$, a sequence of points along the first-order $A-C_{d S}$ transition line is considered. The aim is to investigate the behaviour of two different notions of an (effective) lattice spacing as a function on phase space. Comparing measurements at different points in phase space is of course not meaningful a priori unless they lie on the same renormalization group trajectory of constant physics, as described in Sec. 6.4 above (see also 93 for related comments) ${ }^{26}$ The paper [92] illustrates some of the issues that arise in the absence of a full-fledged renormalization group analysis. Its first proposition is to extract what the authors call an "absolute lattice spacing" from measuring the quantum fluctuations around de Sitter space, as introduced in [13, 14]. A difficulty here is the fact that the effective action for the spatial volume at the $A$ -

\footnotetext{
${ }^{26}$ Moving along such a trajectory towards a UV fixed point, the lattice spacing $a$ should vanish.
} 


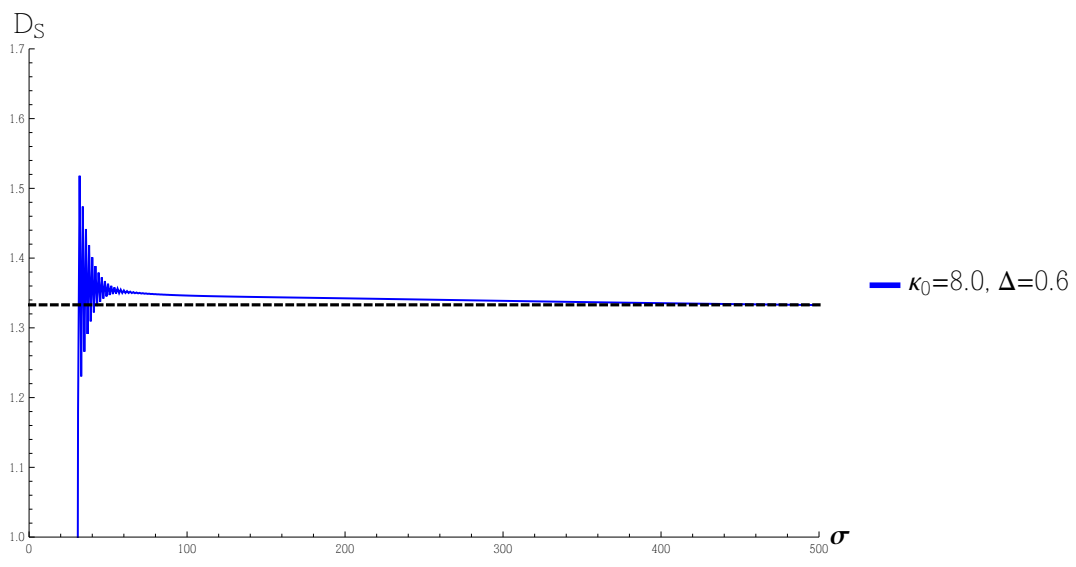

Figure 18: The spectral dimension $D_{S}$ of spacetime in phase $A$, measured at $\left(\kappa_{0}, \Delta\right)=(8.0,0.6)$, for $t_{t o t}=80$ and $N_{41}=160 k$, exhibits a fractal, scale-independent behaviour. [Figure from [91].]

$C_{d S}$ transition is very different from that inside phase $C_{d S}$, because the kinematic term vanishes, as described in Sec. 6.1. To what extent a de Sitter description still captures this situation adequately is unclear. In addition, [92] shows that different ways of accounting for the asymmetry between the numbers of $(4,1)$ - and $(3,2)$-simplices, which depends on the location in phase space, lead to significant differences for the measurements of the lattice spacing for points away from the $A-C_{d S}$ transition.

The second proposition of 92 is to extract a "relative lattice spacing" $a_{r e l}$ by fitting spectral dimension curves at different points in phase space, using a fit function

$$
D_{S}(\sigma)=a-b /\left(c+\sigma / a_{r e l}^{2}\right)
$$

where $a, b$ and $c$ are constants and $a_{r e l}$ is adjusted to achieve a best overlap between different curves. Here, the difficulties already faced in [91] come to a head when trying to measure the spectral dimension very close to the $A-C$ transition. The curves become extremely flat, with $D_{S}$ barely exceeding 1.6 at the upper end $\sigma=500$ of the $\sigma$-range considered. Consequently, the curves have to undergo a very large rescaling compared to the curve at the reference point $\left(\kappa_{0}, \Delta\right)=(2.2,0.6)$, leading to extremely small ranges in the rescaled $\sigma$ available for fitting. One has to set $a \approx 4$ by hand in eq. (32) to be able to make any comparison between the curves. - The systematic and theoretical uncertainties 
just described make it difficult to judge whether the qualitative increase in the lattice spacing for growing coupling $\kappa_{0}$ (at fixed $\Delta=0.6$ ) observed in $[91,92]$ is a genuine physical effect, which would imply that these simulations probe closer into the Planckian regime. A resolution of these issues will most likely require a larger set of observables and a better understanding of the behaviour of CDT quantum gravity under renormalization.

An idea first suggested in the context of three-dimensional CDT [90] is to consider the entire spectral dimension function $D_{S}(\sigma)$, not just for small $\sigma$, to learn more about quantum geometry and its classical limit. An obvious difficulty with this proposal is that, even classically, away from the asymptotic regimes $\sigma \rightarrow 0$ and $\sigma \rightarrow \infty, D_{S}(\sigma)$ contains information about the curvature, shape and topology of a space in a rather summary and integral manner, such that thinking of $D_{S}$ as an effective dimension is in most instances not particularly meaningful. Evaluating $D_{S}$ on ensembles of triangulated manifolds adds finite-size effects, discretization artefact: ${ }^{27}$ and genuine quantum effects to the mix, in a way that in general will be difficult to disentangle, certainly at the lattice volumes currently within reach. Similarly, the suggestion of extracting absolute physical scales from the behaviour of $D_{S}(\sigma)$ for the purpose of defining renormalization group flows [87] seems difficult to realize: the relatively robust short-scale dimension does not refer to any sharply defined transition point to classicality, while the universal nature of other features of the spectral dimension function at intermediate scales (like its maximum) are not well understood either.

Noteworthy in the context of spectral observables is a recent attempt to tap into the potentially rich information contained in the eigenvalues and eigenvectors of the Laplace-Beltrami operator on spatial slices with topology $S^{3}$ in CDT [50]. Which properties of the spectrum lend themselves to ensemble averaging and are ultimately related to geometric observables in the continuum is largely unexplored territory. The fractal and largely nonclassical geometric nature of the slices of constant proper time has been exhibited previously [11,95]. The authors of [50] solve the eigensystem for the Laplacian associated with the four-valent graphs dual to spatial triangulations for sample points in all four phases of CDT, searching for typical features of the spectrum that characterize and distinguish between the different phases, and for new order parameters to help analyze the nature of the phase transition lines. The simulations were performed at fixed volumes $N_{41} \leq 80 \mathrm{k}$ and for $t_{\text {tot }}=80$, and a range of discrete spatial volumes $V_{S}$ of up to about 3000 in terms of the number of tetrahedra.

Since the eigenvalues $\lambda_{n}, n=0,1,2, \ldots$, can be ordered according to size, with $\lambda_{0}=0$ and $\lambda_{1}$ denoting the smallest nonvanishing eigenvalue, one can for example monitor the distribution of a particular eigenvalue with label $n_{0}$, where

\footnotetext{
${ }^{27}$ see 94 for examples of discretization effects on some regular lattices
} 


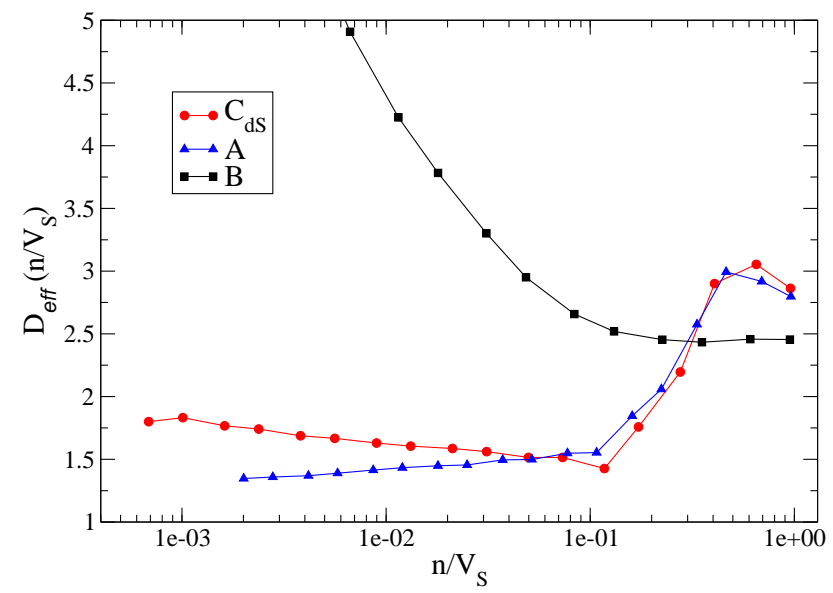

Figure 19: Effective spectral dimension $D_{\text {eff }}$ of spatial slices at $\left(\kappa_{0}, \Delta\right)=(5.0,0.6)$ in phase $A,(2.2,-0.2)$ in phase $B$ and $(2.2,0.6)$ in phase $C_{d S}$, measured for $t_{\text {tot }}=80$, spacetime volume $N_{41}=80 \mathrm{k}$ (phases $A$ and $C_{d S}$ ) and $N_{41}=16 \mathrm{k}$ (phase $B$ ), and in small bins of $n / V_{S}[50]$. In the kinematical limit $n / V_{S} \rightarrow 1$ the effective dimensions are close to 3 , primarily determined by the discrete lattice set-up. [Figure from [50].]

the outcome will in general depend on both the location in phase space and the spatial volume of the slice. When measuring the density $\rho(\lambda)$ of the first 100 eigenvalues for different spatial volumes, [50] found a completely different behaviour in phases $B$ and $C_{d S}$. While in phase $B$ there is a gap $\Delta \lambda=\lambda_{1} \approx 0.1$ that stays practically constant when the slice volume is varied, there is no such gap in the de Sitter phase, where instead $\lambda_{1}$ converges to zero in the thermodynamic limit 28 The former is characteristic for a highly connected space or graph with large Hausdorff dimension and small diameter, and is consistent with what is already known about the geometry of phase $B$. By contrast, in phase $C_{d S}$ one finds a behaviour compatible with Weyl's law for the asymptotic scaling of $n(\lambda)$ - the number of eigenvalues below the value $\lambda$ - for large $\lambda$, namely,

$$
n(\lambda \rightarrow \infty) \propto V \lambda^{d / 2} /(2 \pi)^{d},
$$

valid on a $d$-dimensional smooth space of volume $V$. In analogy with the earlier described method of extracting a spectral dimension from the return probability of a random walker on a piecewise flat space by generalising the smooth-space

\footnotetext{
${ }^{28}$ Both of these have been confirmed in a more detailed follow-up study [96. Interestingly, the expectation values $\left\langle\lambda_{n}\right\rangle$ of low-lying eigenvalues in phase $B$ seem to approach a discrete set of distinct values in the infinite-volume limit.
} 
relation $(30)$, the relation $(33)$ can be used to extract an effective spectral dimension

$$
D_{e f f}:=2 \frac{d \log n / V_{S}}{d \log \lambda_{n}}
$$

for nonsmooth spaces. In this context, considering the large- $\lambda$ limit is the analogue of considering the small- $\sigma$ regime of the random walker. Beyond very short distances, which are dominated by lattice artefacts, the spectral dimension determined in this way is $D_{\text {eff }} \approx 1.5$ in phase $C_{d S}$, in agreement with earlier measurements using the return probability [11,97]. As illustrated by Fig. 19, the same approximately constant value is found in phase $A$, whereas in phase $B$ one

finds a strongly increasing $D_{\text {eff }}$ for decreasing $n / V_{S}$, compatible with an infinite spectral dimension.

In line with the properties of the bifurcation phase discussed earlier, the spectral analysis in this phase reveals characteristic phase- $B$ and phase- $C_{d S}$ behaviour on alternating spatial slices. For the slices of $B$-type this implies the existence of a spectral gap $\lambda_{1}$ and a general shift to larger values for eigenvalues $\lambda_{n}$ of the same order $n$. The jumps between large and small values are particularly apparent for the low-lying part of the spectrum, and become somewhat less pronounced as $n$ increases. The suggestion in [50], further elaborated in [96], is to approach the $C_{b}-C_{d S}$ phase transition from inside the bifurcation phase $C_{b}$, and use the discrete set of eigenvalues $\left\langle\lambda_{n}\right\rangle$ associated with the slices of type $B$, which one expects to vanish as the transition line is approached, as order parameters. In the exploratory study [96], the coupling $\kappa_{0}$ is kept fixed as $\Delta$ is increased towards the phase transition. The critical values found for $\Delta$ are consistent with those found for other order parameters described in Sec. 6.1 above, but the size of the simulations does not yet allow for a reliable measurement of critical exponents, or for a verification that the spectral gaps do indeed vanish at the transition.

To summarize, although there clearly is a lot of (fine-grained) information contained in the Laplacian spectrum of ensembles of causal dynamical triangulations, it remains a challenge to extract from it observables - beyond the relatively well-understood short-scale spectral dimension - which describe genuine, universal quantum properties of spacetime, and upon coarse-graining relate to (quasi-)local semi-classical geometric features.

\subsection{Curvature observables}

Given that curvature is such a central notion in classical general relativity, one may wonder what role it has to play in nonperturbative quantum gravity. There are multiple obstacles in representing curvature in the quantum theory, which have hampered developments so far. However, there has been a recent and promising breakthrough in defining a notion of Ricci curvature in CDT, which we will 
describe later in this section. One reason why the need for quantum curvature may not appear as pressing is the existence of a straightforward prescription for the curvature scalar $R(x)$ in Regge calculus in terms of deficit angles [35], which is also used in representing the Einstein-Hilbert action (3) in CDT quantum gravity, see [17] for a derivation. Of course, one needs to keep in mind that the curvature scalar, like any other local curvature invariant, is not a good observable unless it is integrated over spacetime, say.

However, even the integrated form of the curvature scalar turns out to be not well suited as a quantum observable. Not unexpectedly for the counterpart of a second-order differential operator in the continuum, the total Regge scalar curvature diverges in the continuum limit, but it is not clear how to renormalize it appropriately. Put differently, the deficit angle prescription is associated with the cut-off scale and there is no obvious way of scaling it up, in the sense of associating "effective" deficit angles to coarse-grained regions of spacetime. Another shortcoming is the fact that the curvature scalar captures only a small part of the information contained in the full Riemann tensor. It is certainly possible to construct finite-difference expressions that represent the Riemann tensor on piecewise flat manifolds, but they inevitably would be unwieldy and highly nonunique, and - to the extent that they are still based on deficit angles representing the Gaussian curvature of two-dimensional subspaces - would suffer from the same problems as the scalar curvature.

To ameliorate the singular nature of curvature in the quantum realm and realize gauge invariance at the same time, an old idea coming from gauge field theory is to capture curvature information in terms of nonlocal Wilson loop observables, obtained by integrating the associated gauge connection along one-dimensional closed curves in spacetime. Similarly, in gravity one can consider the gravitational Wilson loop $W_{\gamma}$ associated with a loop $\gamma$ on a spacetime manifold $M$ with metric $g_{\mu \nu}$ and associated Levi-Civita connection $\Gamma_{\mu \nu}^{\lambda}$, as the trace of the path-ordered exponential of $\Gamma$ along $\gamma$,

$$
W_{\gamma}[\Gamma]:=\operatorname{Tr} \mathcal{P} \exp \left(-\oint_{\gamma} \Gamma\right)
$$

The path ordering, indicated by $\mathcal{P}$, is needed because of the nonabelian nature of the connection. The expression under the trace in (35) is a $S O(3,1)$-valued functional, a so-called "holonomy", acting on tangent vectors at the base point $p \in M$ where the loop $\gamma$ begins and ends. (The analogous quantity in the Wickrotated CDT setting will be $S O(4)$-valued.) Unlike in gauge theory, Wilson loops in pure gravity are not observables, because they refer to a closed curve $\gamma$ in spacetime and therefore are not diffeomorphism-invariant. There are different ways of constructing observables from Wilson loops, for example by "marking" the location of the loop in terms of matter degrees of freedom or by performing 
averages over loops or subsets of loops that share certain invariant geometric features regarding their length and shape.

These considerations are relevant for CDT, where parallel transport and Wilson loops can be defined in a straightforward way, as described in [43]. Because of the piecewise flat nature of the geometry this turns out to be far easier than on a smooth curved manifold. A natural prescription is to use straight path segments between the centres of pairs of adjacent four-simplices and consider the holonomies of piecewise straight loops made from such segments. To compute the path-ordered exponentials requires the introduction of a coordinate system 29 in each simplex at an intermediate stage of the calculation, which drops out again upon taking the trace.

On a smooth four-dimensional Riemannian manifold, the gravitational Wilson loop of an infinitesimal square loop $\gamma_{[\mu \nu]}$ of geodesic edge length $\varepsilon$ in the $\mu-\nu$ plane depends to lowest nontrivial order on the entries $R_{. \mu \nu}$ of the Riemann curvature tensor according to

$$
W_{\gamma_{[\mu \nu]}}=4+\varepsilon^{4} R_{\lambda \mu \nu}^{\kappa} R_{\kappa \mu \nu}^{\lambda}+\mathcal{O}\left(\varepsilon^{5}\right)
$$

where the 4 comes from the trace of the unit matrix in the defining representation of $S O(4)$ (see, for example, [98]). On a general curved manifold, there is no obvious way to relate the Wilson loop of a noninfinitesimal loop to some (integrated or coarse-grained) form of local curvature in a similar way ${ }^{30}$ However, it is a priori conceivable that in nonperturbative quantum gravity like CDT, Wilson loops which probe the quantum geometry on scales sufficiently large compared to the Planck scale but are still infinitesimal from a classical point of view display a semiclassical behaviour, where quantum fluctuations mostly "average out", leading to expectation values for the Wilson loops close to (4 times) the identity, with small deviations that characterize some effective curvature scale.

This hypothesis was investigated in simulations of four-dimensional CDT with spherical slices at $\left(\kappa_{0}, \Delta\right)=(2.2,0.6)$, for $t_{\text {tot }}=80$ and $N_{4}=20 k$ and loops of winding number 1 with respect to the compactified time direction [43. To obtain a genuine Wilson loop observable, the underlying loop $\gamma$ was identified with the spacetime trajectory of a massive particle moving forward in time along one of the piecewise straight paths mentioned earlier. The total action of the system consists of the usual Einstein-Hilbert piece and an extra term $m_{0} \ell(\gamma)$ proportional to the discrete length $\ell$ of the loop, and its partition function is a double sum over triangulated geometries and nonselfintersecting paths $\gamma$. The observable extracted from the Wilson loop measurements was the expectation value of the probability distribution $P\left(\theta_{1}, \theta_{2}\right)$ of two invariant angles $\theta_{i} \in[0, \pi]$ that characterize elements

\footnotetext{
${ }^{29}$ Barycentric coordinates are a convenient choice 43 .

${ }^{30} \mathrm{~A}$ gravitational version of the nonabelian Stokes theorem is unlikely to be useful because curvature appears only inside a complicated, nonlocal expression involving area ordering 99].
} 


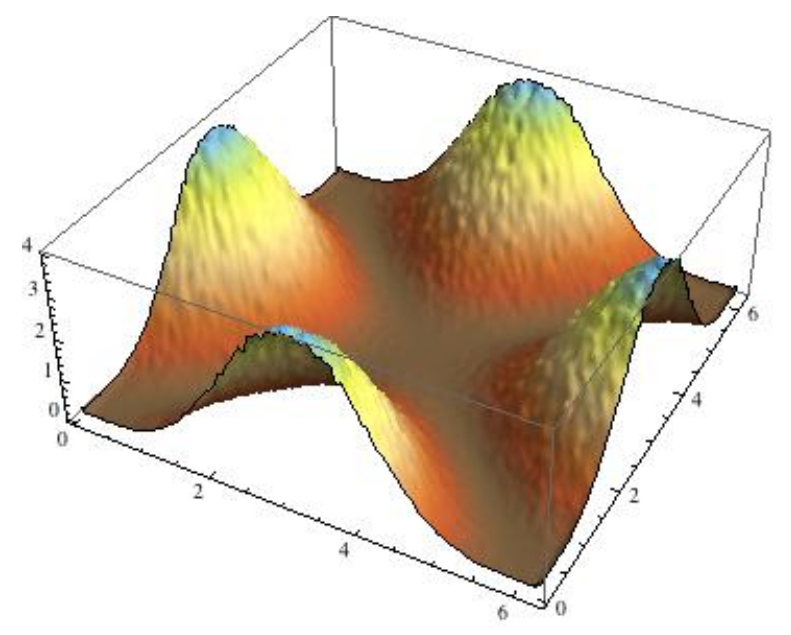

Figure 20: Histogram of the invariant angles $\left(\theta_{1}, \theta_{2}\right)$, extracted from Monte Carlo measurements of Wilson loops in CDT [43], matching the functional form (37) up to an overall normalization.

of $S O(4)$ up to conjugation. It is straightforward to compute $P\left(\theta_{1}, \theta_{2}\right)$ analytically for the case that the holonomies are uniformly distributed with respect to the Haar measure on the group manifold of $S O(4)$. The normalized distribution is given by

$$
P\left(\theta_{1}, \theta_{2}\right)=\frac{1}{\pi^{2}} \sin ^{2}\left(\frac{\theta_{1}+\theta_{2}}{2}\right) \sin ^{2}\left(\frac{\theta_{1}-\theta_{2}}{2}\right) .
$$

It turns out that for a wide range of bare masses $m_{0}$, the distributions measured in the CDT simulations match this functional form perfectly (see Fig. 20), from which several conclusions may be drawn. On the one hand, there are no obvious discretization effects connected to the fact that (for the four-dimensional equilateral simplices used) all deficit angles come in integer units of $\arccos (1 / 4)$, the angle between two three-dimensional faces sharing a two-dimensional triangle. On the other hand, there is no sign that for the ensemble of loops under consideration the Wilson loops cluster around the identity $\left(\theta_{1}, \theta_{2}\right)=(0,0)$, which would be a potential indicator of an averaging-out of small-scale fluctuations of the curvature. It could mean that the loops considered here are too large - their minimal discrete length is $\ell=360$, due to the way the dual paths move between adjacent spatial layers. This is a hypothesis that could be tested relatively easily. Alternatively, there may be more subtle ways of extracting interesting geometric information from Wilson loops, perhaps from higher-order correlations. It is also possible that Wilson loops are simply not suited as a measure of coarse-grained local curvature in quantum gravity, because they are still too singular.

The Wilson loop investigation just described provides another motivation for 


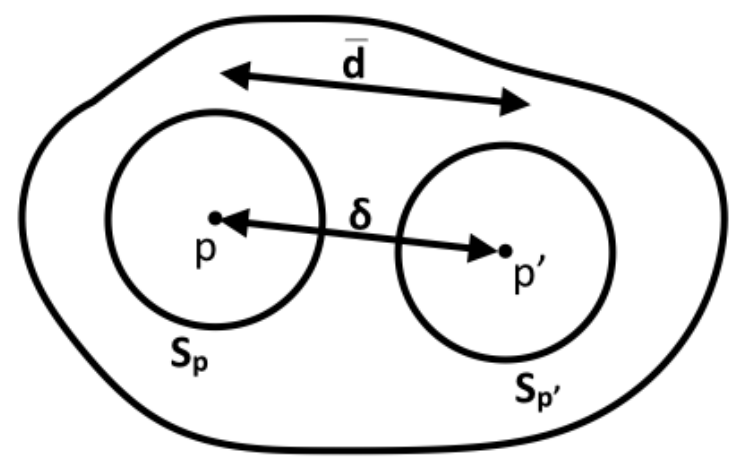

Figure 21: To obtain the local Ricci curvature $\operatorname{Ric}(v, v)$ associated with a vector $v$ on a smooth Riemannian space, one compares the distance $\bar{d}$ of two small nearby spheres $S_{p}$ and $S_{p^{\prime}}$ with the distance $\delta=|v|$ of their centres $p$ and $p^{\prime}$, measured along the unique geodesic from $p$ to $p^{\prime}$ in the direction of $v$.

finding more useful curvature observables with better averaging properties. The recent suggestion of defining a scalable notion of quantum Ricci curvature [100] without referring to deficit angles is a promising step in this direction, which has already been tested for dynamical triangulations in lower dimensions [101]. The key idea, inspired by a classical characterization of curvature on smooth $D$-dimensional Riemannian spaces and later generalized to general metric spaces [102], is to compare the distance $\bar{d}$ of two $(D-1)$-spheres with the distance $\delta$ between their centres (see Fig. 21). More specifically, on a Riemannian space with positive (negative) Ricci curvature, the distance $\bar{d}\left(S_{p}, S_{p^{\prime}}\right)$ of two small nearby spheres $S_{p}$ and $S_{p^{\prime}}$ is smaller (bigger) than the distance $\delta$.

The quantum Ricci curvature defined in 100 and evaluated on a variety of piecewise flat spaces, including regular lattices and Delaunay triangulations, uses a variant where the sphere radii are both set equal to the centre distance $\delta$. In addition, the sphere distance is defined as the average distance of $S_{p}^{\delta}$ and $S_{p^{\prime}}^{\delta}$,

$$
\bar{d}\left(S_{p}^{\delta}, S_{p^{\prime}}^{\delta}\right):=\frac{1}{\operatorname{vol}\left(S_{p}^{\delta}\right)} \frac{1}{\operatorname{vol}\left(S_{p^{\prime}}^{\delta}\right)} \int_{S_{p}^{\delta}} d^{D-1} q \sqrt{h} \int_{S_{p^{\prime}}^{\delta}} d^{D-1} q^{\prime} \sqrt{h^{\prime}} d\left(q, q^{\prime}\right),
$$

or a suitable analogue of this prescription on the metric space in question. In (38), $h$ and $h^{\prime}$ are the determinants of the metrics induced on the two $(D-1)$ dimensional "spheres" 31 , which are also used to compute the sphere volumes $\operatorname{vol}(S)$, and $d\left(q, q^{\prime}\right)$ denotes the geodesic distance between the points $q$ and $q^{\prime}$.

\footnotetext{
${ }^{31}$ Note that when using this construction for macroscopic scales $\delta$, the sphere $S_{p}^{\delta}$ is defined as the set of all points at geodesic distance $\delta$ from the point $p$, and will in general not have the topology of an $S^{D-1}$-sphere.
} 


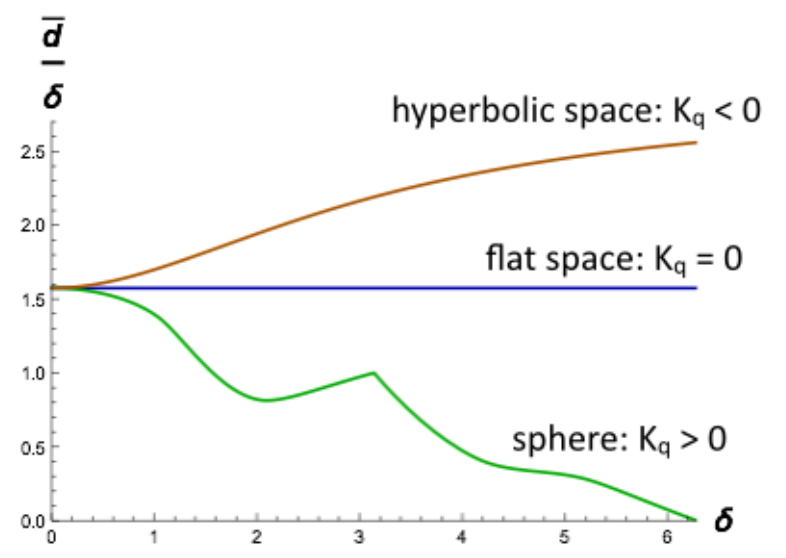

Figure 22: The normalized average sphere distance $\bar{d} / \delta$ as a function of $\delta \in[0,2 \pi]$ for three constantly curved model spaces in two dimensions. The analogous curves in higher dimensions look qualitatively similar. The curvature radius has been set to 1 wherever applicable.

The presence of a single linear distance scale $\delta$ then allows us to define a scalable notion of quantum Ricci curvature $K_{q}$ at the scale $\delta$ from the quotient

$$
\frac{\bar{d}\left(S_{p}^{\delta}, S_{p^{\prime}}^{\delta}\right)}{\delta}=c_{q}\left(1-K_{q}\left(p, p^{\prime}\right)\right), \quad \delta=d\left(p, p^{\prime}\right),
$$

where $c_{q}$ is a positive constant with $0<c_{q}<3$, which depends on the metric space under consideration, and $K_{q}$ captures any nontrivial dependence on $\delta$. The beauty of this prescription is that it relies only on being able to measure geodesic distances and volumes, both of which are easily accessible in (C)DT, and that it captures directional, tensorial information. Part of the strategy when measuring quantum Ricci curvature in CDT quantum gravity will be to try and understand whether in any range of scales its behaviour resembles that of a classical curved space. An important point of comparison are the curves for the normalized average sphere distance $\bar{d} / \delta$ for constantly curved smooth spaces (with positive, negative and vanishing curvature) in any dimension, which can be computed analytically (c.f. Fig. 22). A first indication of the robustness of the quantum Ricci curvature comes from evaluating it on the ensemble of Euclidean dynamical triangulations in two dimensions [101], which is known to have a highly nonclassical and fractal geometry. The measured expectation value of its curvature can be mapped best to that of a five-dimensional continuum sphere, where it should be recalled that Euclidean quantum gravity in two dimensions has a Hausdorff dimension of four (which at least comes close). Measurements of the quantum 
Ricci curvature in CDT in four dimensions are under way [103], and will for the first time give an indication to what extent the dynamically generated de Sitter universe - identified on the basis of its large-scale dimension and volume profile - resembles a de Sitter space also in terms of its quasi-local curvature properties.

\section{Prospects for cosmology}

On the basis of the quantum observables known and measured in CDT quantum gravity so far, what is the status of extracting physical predictions, without invoking additional ad hoc assumptions? At this stage, no statements of substance have been made about specific, quantitative predictions of new physics due to genuine quantum gravity effects. However, it should be noted that $C D T$ predicts a positive (renormalized) cosmological constant $\Lambda$. This feature of dynamically triangulated models has to do with the convergence requirement of the nonperturbative path integral and the renormalization behaviour of the cosmological constant in the continuum limit. CDT does not predict any particular value for the physical $\Lambda$ per se, only that it should be positive, which is of course in agreement with our current understanding of the universe based on $\Lambda \mathrm{CDM}$ cosmology.

The most obvious place to look for physical implications is early universe cosmology. In this context, it is encouraging that CDT has been shown to reproduce several aspects of the classical theory, which is not the norm in nonperturbative quantum gravity, as pointed out earlier. The matching with a semiclassical behaviour of the de Sitter volume profile including quantum fluctuations for a universe of only about 20 Planck lengths across 13, 14 is remarkable, and an indication that - at least for selected observables - the scale gap from Planckian to semiclassical is perhaps less daunting than it appears naïvely. Nevertheless, the observables currently accessible in CDT quantum gravity are still too few and too coarse to allow for a more detailed comparison.

A next step in the development of the quantum theory is therefore to ascertain whether and on what scale the dynamically generated de Sitter universe can be said to resemble a smooth, constantly curved de Sitter space in terms of its local geometric properties, beyond displaying the correct large-scale dimensionality and volume profile. Conversely, what would be desirable on the cosmology side is the formulation of explicitly diffeomorphism-invariant and background-independent observables. Because of the vastly different languages used in the full quantum theory (nonperturbative, nonsmooth, no fixed background, manifest diffeomorphism invariance) and in standard cosmology (perturbative, smooth, cosmological background metric, gauge-fixed coordinates), such observables constitute the natural common ground where the two formulations can be compared. 


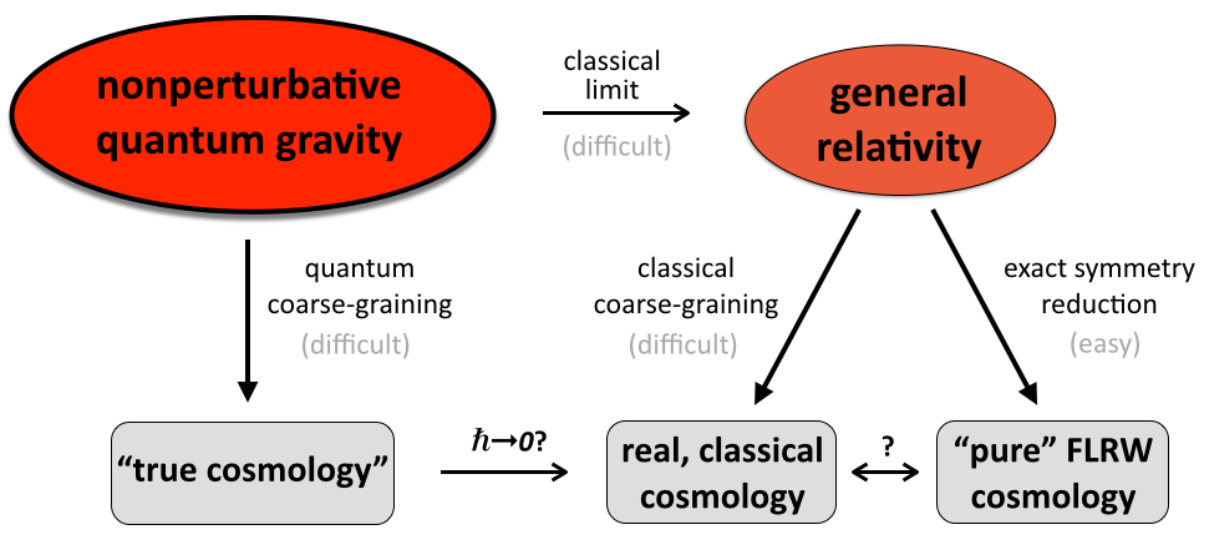

Figure 23: Relations in theory space: different ways to derive cosmology from nonperturbative quantum gravity and general relativity.

Let us emphasize that all CDT results discussed so far were extracted without making assumptions about the homogeneity or isotropy of spacetime at the outset. For instance, the expectation value of the volume profile for spacetimes of topology $S^{1} \times S^{3}$ was obtained by integrating over all geometric modes in the path integral, without resorting to any a priori symmetry reduction or a symmetryrelated truncation of the degrees of freedom. Remarkably, it then turned out that in an extended region of phase space the outcome can be interpreted in terms of an isotropic and homogeneous solution of the Einstein equations.

Of course, given a fundamental, nonperturbative theory of quantum gravity, all quantum and classical aspects of gravity and cosmology must ultimately follow from it, including the quantum behaviour of the very early universe and finding the nonperturbative quantum ground state of the universe, if it exists. It is an interesting question whether there are observable aspects of large-scale cosmology today that can only be understood in terms of a full-fledged theory of quantum gravity, and not classical general relativity (or perhaps quantum cosmology) alone. To exhibit phenomenological consequences in cosmology or elsewhere is of course one of the raisons d'être of quantum gravity.

Fig. 23 illustrates various ways of arriving at cosmology from either quantum or classical gravity, assuming that general relativity is the correct theory classically. By "true cosmology" we mean all large-scale phenomena derived directly from nonperturbative quantum gravity by integrating out or coarse-graining its microscopic degrees of freedom. It should be contrasted with standard quantum cosmology, which denotes a quantum-mechanical treatment of only those degrees of freedom that are left after a classical symmetry reduction ${ }^{32}$, that is, a quantum

\footnotetext{
${ }^{32}$ In the gravitational sector of Friedmann-Lemaître-Robertson-Walker (FLRW) cosmologies,
} 
version of the "pure FLRW cosmology" in the bottom right-hand corner of Fig. 23. Readers interested in further considerations on the relation between CDT and cosmology should consult [104], which also compares the robustness of the classical FLRW paradigm with the fact that in candidate theories of nonperturbative quantum gravity the emergence of a scale factor exhibiting FLRW behaviour is definitely not automatic, unless one adopts causality conditions à la CDT, as explained in Sec. 5 above. Whether and how the two "effective" scale factors and their dynamics are related requires further research. Another interesting question is to what extent quantum coarse-graining, especially for a quantity like the Ricci curvature [100], can shed light on the little-understood coarse-graining properties of the classical Einstein or Friedmann equation ${ }^{33}$ and vice versa.

Generally speaking, looking for observables that can quantify the presence of (approximate) global symmetries at a given scale of a quantum geometry is an important and interesting topic, especially after one has established the presence of an extended geometry, as in the $C$-phases of CDT. It has been suggested to use the variance of the return probability $P(\sigma)$ (discussed in Sec. 7.1) and the variance of the normalized volume of geodesic balls of radius $r$ for a given triangulation $T$ as measures for the homogeneity of the geometry of $T$ [107]. However, a smallscale simulation for CDT in $2+1$ dimensions of the expectation values of these quantities did not produce a conclusive result on whether they can be regarded as meaningful observables in a continuum limit. - Of course, homogeneity can in principle be tested for arbitrary local quantities whose averages give rise to observables, leading us back to a recurring theme of this review, namely, the need for more observables (which by the way is also an issue familiar to cosmologists).

\section{Outlook}

CDT quantum gravity has come a considerable way since its inception as a nonperturbative Lorentzian path integral for gravity [5 7]. While the first big review in 17 gave a thorough account of the ingredients and construction of the model, and described some of CDT's trademark results, the present review documents the next, more mature stage of the theory as a serious contender for nonperturbative quantum gravity. As emphasized in Sec. 2, a characteristic feature of this approach is to take numerical tools seriously as a legitimate way of advancing our quantitative understanding of "bulk quantum gravity" in a Planckian regime, to which we currently do not have analytical access, either by conventional or exotic

this is just the scale factor $a(t)$.

${ }^{33}$ The so-called averaging problem of cosmology refers to the difficulty of quantifying the effect of inhomogeneities on smaller scales on the dynamics of the universe on larger scales, beyond a linear regime, see 105,106] for recent reviews. 
means. A partial exception are functional renormalization group techniques in an asymptotic safety scenario [21,22], although also this ansatz relies crucially on numerical methods. This observation and the methodological complementarity of the two approaches suggests that developing them in tandem may be a fruitful research strategy.

Structurally, the parallels between CDT lattice gravity and lattice gauge theory are coming into sharper focus, despite the obvious differences in terms of their dynamical degrees of freedom and the nature of their gauge symmetries. One beautiful and possibly key aspect of CDT is its manifest diffeomorphism invariance, as spelled out in Sec. 4, which may have been somewhat underappreciated in the past. Although realized in a different way, it can be thought of as the gravitational counterpart of the exact gauge invariance of Wilson's timehonoured lattice formulation of QCD [108], which is also preserved, despite the lattice discretization. Apart from the enormous technical convenience of not having to deal with coordinate redundancy, this also implies that the physical and geometric content of CDT are not obscured by gauge issues.

In complete analogy with lattice gauge field theory, computational resources are a limiting factor, and finding ever better ways of addressing standard issues of lattice Monte Carlo simulations (such as finite-volume effects, lattice artefacts, statistical errors, numerical efficiency and critical slowing down) will be a continuing challenge. However, because of the dynamical nature of gravity - translated into the dynamical nature of the triangulated lattices of CDT - and the absence of a fixed background reference frame, many of these issues come with a new and interesting twist. The ensuing subtleties were illustrated well by our discussion in Sec. 6.4 about defining renormalization group trajectories of constant physics without a predefined notion of scale. Progress in CDT provides a blueprint for how these difficulties, which are not present in quantum field theory on a fixed background, may be addressed and overcome.

There is a clear road ahead for CDT quantum gravity, with plenty of interesting research problems to tackle, many of which have already been mentioned throughout this review. They are still mostly of a fundamental nature, but include technical challenges of implementability and measurability. Analyzing the observables described in Sec. 7 in detail and coming up with new ones is a rich playground for creative minds and will play an important role in addressing key open issues. These include (i) getting a better handle on the renormalization group analysis, thus paving the way toward verifying the asymptotic safety conjecture; (ii) obtaining a more detailed understanding of the properties of quantum spacetime near the Planck scale, especially in the vicinity of the second-order phase transitions, and fleshing out the nature of any UV completion; (iii) investigating the physical nature of the new bifurcation phase and the mechanism behind the $C_{b^{-}} C_{d S}$ phase transition; (iv) getting a better theoretical understanding of how 
to couple matter and relate it in a meaningful way to semiclassical observables; (v) further strengthening the evidence for a well-defined classical limit and quantifying quantum deviations from it; and (vi) creating a bridge toward cosmology and coming up with signatures of quantum gravity phenomenology.

It is fortunate that CDT quantum gravity comes with a theoretical framework and computational tools that allow us to address these questions directly. It would be even more remarkable if in a few years' time from now we would find ourselves in a situation where CDT is no longer regarded as a candidate theory of quantum gravity, but "merely" as a powerful method that enables us to understand and quantify quantum gravity nonperturbatively, in the same way as lattice gauge theory has given us unique access to the nonperturbative properties of quantum chromodynamics.

\section{Acknowledgments}

It is a pleasure to thank J. Ambjørn, A. Görlich and J. Jurkiewicz for longstanding collaboration and for their feedback on the manuscript. - This research was supported in part by Perimeter Institute for Theoretical Physics. Research at Perimeter Institute is supported by the Government of Canada through the Department of Innovation, Science and Economic Development and by the Province of Ontario through the Ministry of Research and Innovation.

\section{References}

[1] J. Ambjørn and J. Jurkiewicz, Four-dimensional simplicial quantum gravity, Phys. Lett. B 278 (1992) 42-50.

[2] R. Loll, Discrete approaches to quantum gravity in four dimensions, Living Rev. Rel. 1 (1998) 13 [arXiv: gr-qc/9805049], ch.4.

[3] M. Rocek and R.M. Williams, Quantum Regge calculus, Phys. Lett. 104B (1981) 31-37.

[4] H.W. Hamber, Quantum gravity on the lattice, Gen. Rel. Grav. 41 (2009) 817-876 arXiv:0901.0964, gr-qc].

[5] J. Ambjørn and R. Loll, Non-perturbative Lorentzian quantum gravity, causality and topology change, Nucl. Phys. B 536 (1998) 407-434 [arXiv: hep-th/9805108]. 
[6] J. Ambjørn, J. Jurkiewicz and R. Loll, A non-perturbative Lorentzian path integral for gravity, Phys. Rev. Lett. 85 (2000) 924-927 [arXiv: hepth/0002050].

[7] J. Ambjørn, J. Jurkiewicz and R. Loll, Dynamically triangulating Lorentzian quantum gravity, Nucl. Phys. B 610 (2001) 347-382 [arXiv: hep-th/0105267].

[8] J. Ambjørn, J. Jurkiewicz and R. Loll, Emergence of a $4 D$ world from causal quantum gravity, Phys. Rev. Lett. 93 (2004) 131301 [arXiv: hep-th/0404156].

[9] J. Ambjørn, J. Jurkiewicz and R. Loll, Semiclassical universe from first principles, Phys. Lett. B 607 (2005) 205-213 [arXiv: hep-th/0411152].

[10] J. Ambjørn, J. Jurkiewicz and R. Loll, Spectral dimension of the universe, Phys. Rev. Lett. 95 (2005) 171301 [arXiv: hep-th/0505113].

[11] J. Ambjørn, J. Jurkiewicz and R. Loll, Reconstructing the universe, Phys. Rev. D 72 (2005) 064014 [arXiv: hep-th/0505154].

[12] S. Carlip, Dimension and dimensional reduction in quantum gravity, Class. Quant. Grav. 34 (2017) 193001 arXiv:1705.05417, gr-qc]; Universe 5 (2019) 83 arXiv:1904.04379, gr-qc].

[13] J. Ambjørn, A. Görlich, J. Jurkiewicz and R. Loll, Planckian birth of the quantum de Sitter universe, Phys. Rev. Lett. 100 (2008) 091304 arXiv:0712.2485, hep-th].

[14] J. Ambjørn, A. Görlich, J. Jurkiewicz and R. Loll, The nonperturbative quantum de Sitter universe, Phys. Rev. D 78 (2008) 063544 arXiv:0807.4481, hep-th].

[15] J. Ambjørn, S. Jordan, J. Jurkiewicz, and R. Loll, Second-order phase transition in Causal Dynamical Triangulations, Phys. Rev. Lett. 107 (2011) 211303 arXiv:1108.3932, hep-th].

[16] J. Ambjørn, S. Jordan, J. Jurkiewicz, and R. Loll, Second- and first-order phase transitions in causal dynamical triangulations, Phys. Rev. D 85 (2012) 124044 [arXiv:1205.1229, hep-th].

[17] J. Ambjørn, A. Görlich, J. Jurkiewicz and R. Loll, Nonperturbative quantum gravity, Phys. Rept. 519 (2012) 127-210 arXiv:1203.3591, hep-th].

[18] A. Ashtekar and V. Petkov (Eds.), Springer handbook of spacetime (Springer, Berlin, Heidelberg, 2014). 
[19] G. Ellis, J. Murugan and A. Weltman (Eds.), Foundations of space and time (Cambridge University Press, Cambridge, 2012).

[20] D. Oriti (Ed.), Approaches to quantum gravity (Cambridge University Press, Cambridge, 2009).

[21] M. Reuter and F. Saueressig, Quantum gravity and the functional renormalization group (Cambridge University Press, Cambridge, 2019).

[22] R. Percacci, An introduction to covariant quantum gravity and asymptotic safety (World Scientific, Singapore, 2017).

[23] M. Niedermaier, The asymptotic safety scenario in quantum gravity: an introduction, Class. Quant. Grav. 24 (2007) R171 [arXiv: gr-qc/0610018].

[24] M. Reuter and F. Saueressig, Fractal space-times under the microscope: a renormalization group view on Monte Carlo data, JHEP 1112 (2011) 012 arXiv:1110.5224, hep-th].

[25] S. Rechenberger and F. Saueressig, The $R^{2}$ phase-diagram of QEG and its spectral dimension, Phys. Rev. D 86 (2012) 024018 [arXiv:1206.0657, hep-th].

[26] B. Knorr and F. Saueressig, Towards reconstructing the quantum effective action of gravity, Phys. Rev. Lett. 121 (2018) 161304 arXiv:1804.03846, hep-th].

[27] F. David, Simplicial quantum gravity and random lattices, in Gravitation and quantizations. Proceedings Les Houches summer school 1992., eds. J. ZinnJustin and B. Julia (Elsevier, Amsterdam 1995) [arXiv: hep-th/9303127].

[28] J. Ambjørn, B. Durhuus and T. Jonsson, Quantum geometry (Cambridge University Press, Cambridge, 1997).

[29] J. Ambjørn, R. Loll, J.L. Nielsen and J. Rolf, Euclidean and Lorentzian quantum gravity: Lessons from two-dimensions, Chaos Solitons Fractals 10 (1999) 177-195 [arXiv: hep-th/9806241].

[30] J. Ambjørn, A. Görlich, J. Jurkiewicz and R. Loll, Quantum gravity via Causal Dynamical Triangulations, in Springer handbook of spacetime, eds. A. Ashtekar and V. Petkov (Springer, Berlin, Heidelberg, 2014) 723-741 arXiv:1302.2173, hep-th].

[31] A. Görlich, Introduction to Causal Dynamical Triangulations, in Quantum gravity and quantum cosmology, eds. G. Calcagni, L. Papantonopoulos, G. Siopsis and N. Tsamis, Lecture Notes in Physics, vol. 863 (Springer, Berlin, Heidelberg, 2013) 93-117. 
[32] J. Ambjørn, J. Jurkiewicz and R. Loll, Causal dynamical triangulations and the quest for quantum gravity, in Foundations of space and time, eds. G. Ellis, J. Murugan and A. Weltman (Cambridge University Press, Cambridge, 2012) 321-337 arXiv:1004.0352, hep-th].

[33] R. Loll, The emergence of spacetime or quantum gravity on your desktop, Class. Quant. Grav. 25 (2008) 114006 [arXiv:0711.0273, gr-qc].

[34] J. Ambjørn, J. Jurkiewicz and R. Loll, Quantum gravity, or The art of building spacetime, in Approaches to quantum gravity, ed. D. Oriti (Cambridge University Press, Cambridge, 2009) 341-359 [arXiv: hep-th/0604212].

[35] T. Regge, General relativity without coordinates, Nuovo Cim. 19 (1961) 558571.

[36] J. Ambjørn, K.N. Anagnostopoulos and R. Loll, Crossing the $c=1$ barrier in 2-D Lorentzian quantum gravity, Phys. Rev. D 61 (2000) 044010 [arXiv: hep-lat/9909129].

[37] J. Ambjørn, A.T. Görlich, J. Jurkiewicz and H.-G. Zhang, Pseudo-topological transitions in 2D gravity models coupled to massless scalar fields, Nucl. Phys. B 863 (2012) 421-434 [arXiv:1201.1590, gr-qc].

[38] J. Ambjørn, K.N. Anagnostopoulos and R. Loll, A new perspective on matter coupling in 2d quantum gravity, Phys. Rev. D 60 (1999) 104035 [arXiv: hepth/9904012].

[39] J. Ambjørn, K.N. Anagnostopoulos, R. Loll and I. Pushkina, Shaken, but not stirred - Potts model coupled to quantum gravity, Nucl. Phys. B 807 (2009) 251-264 arXiv:0806.3506, hep-lat].

[40] S. Bilke, Z. Burda, A. Krzywicki, B. Petersson, J. Tabaczek and G. Thorleifsson, 4-D simplicial quantum gravity interacting with gauge matter fields, Phys. Lett. B 418 (1998) 266-272 [arXiv: hep-lat/9710077].

[41] J. Ambjørn, L. Glaser, A. Görlich and J. Jurkiewicz, Euclidian 4d quantum gravity with a non-trivial measure term, JHEP 1310 (2013) 100 arXiv:1307.2270, hep-lat].

[42] I. Khavkine, R. Loll and P. Reska, Coupling a point-like mass to quantum gravity with Causal Dynamical Triangulations, Class. Quant. Grav. 27 (2010) 185025 arXiv:1002.4618, gr-qc]. 
[43] J. Ambjørn, A. Görlich, J. Jurkiewicz and R. Loll, Wilson loops in nonperturbative quantum gravity, Phys. Rev. D 92 (2015) no.2, 024013 arXiv:1504.01065, gr-qc].

[44] M. Hanada, Markov chain Monte Carlo for dummies arXiv:1808.08490, hepth].

[45] R.G. Jha, S. Catterall, D. Schaich and T. Wiseman, Testing the holographic principle using lattice simulations, EPJ Web Conf. 175 (2018) 08004 [arXiv:1710.06398, hep-lat].

[46] W. Cunningham and D. Krioukov, Causal set generator and action computer, Comput. Phys. Commun. 233 (2018) 123-133 [arXiv:1709.03013, gr-qc].

[47] https://www.adamgetchell.org/CDT-plusplus/ and personal communication with A. Getchell

[48] J. Laiho, personal communication

[49] R. Kommu, A validation of Causal Dynamical Triangulations, Class. Quant. Grav. 29 (2012) 105003 arXiv:1110.6875, gr-qc].

[50] G. Clemente and M. D'Elia, Spectrum of the Laplace-Beltrami operator and the phase structure of causal dynamical triangulations, Phys. Rev. D 97 (2018) 124022 arXiv:1804.02294, hep-th].

[51] C. Zahn and U. Kraus, Sector models - A toolkit for teaching general relativity: I. Curved spaces and spacetimes, Eur. J. Phys. 35 (2014) 055020 arXiv:1405.0323, physics.ed-ph].

[52] F. David, Loop equations and nonperturbative effects in two-dimensional quantum gravity, Mod. Phys. Lett. A 5 (1990) 1019-1030.

[53] J. Ambjørn, J. Jurkiewicz and Y.M. Makeenko, Multiloop correlators for two-dimensional quantum gravity, Phys. Lett. B 251 (1990) 517-524.

[54] A. Dasgupta and R. Loll, A proper time cure for the conformal sickness in quantum gravity, Nucl. Phys. B 606 (2001) 357-379 [arXiv: hep-th/0103186].

[55] D. Coumbe and J. Laiho, Exploring Euclidean Dynamical Triangulations with a non-trivial measure term, JHEP 1504 (2015) 028 arXiv:1401.3299, hep-th].

[56] T. Rindlisbacher and P. de Forcrand, Euclidean Dynamical Triangulation revisited: is the phase transition really 1st order? (extended version), JHEP 1505 (2015) 138 [arXiv:1503.03706, hep-lat]. 
[57] R. Loll, Discrete Lorentzian quantum gravity, Nucl. Phys. Proc. Suppl. 94 (2001) 96-107 [arXiv: hep-th/0011194].

[58] R. Loll, The emergence of spacetime or quantum gravity on your desktop, Class. Quant. Grav. 25 (2008) 114006 [arXiv:0711.0273, gr-qc].

[59] L. Andersson, G.J. Galloway and R. Howard, The cosmological time function, Class. Quant. Grav. 15 (1998) 309-322 [arXiv: gr-qc/9709084].

[60] P. Hořava, Quantum gravity at a Lifshitz point, Phys. Rev. D 79 (2009) 084008 [arXiv:0901.3775, hep-th].

[61] C. Anderson, S.J. Carlip, J.H. Cooperman, P. Hořava, R.K. Kommu and P.R. Zulkowski, Quantizing Horava-Lifshitz gravity via Causal Dynamical Triangulations, Phys. Rev. D 85 (2012) 044027 [arXiv:1111.6634, hep-th].

[62] S. Jordan and R. Loll, Causal Dynamical Triangulations without preferred foliation, Phys. Lett. B 724 (2013) 155-159 [arXiv:1305.4582, hep-th].

[63] S. Jordan and R. Loll, De Sitter universe from Causal Dynamical Triangulations without preferred foliation, Phys. Rev. D 88 (2013) 044055 arXiv:1307.5469, hep-th].

[64] S. Jordan, Globally and locally causal dynamical triangulations, PhD Thesis, Radboud University Nijmegen, 2013.

[65] J. Ambjørn, J. Jurkiewicz and R. Loll, Nonperturbative 3-d Lorentzian quantum gravity, Phys. Rev. D 64 (2001) 044011 [arXiv: hep-th/0011276].

[66] R. Loll and B. Ruijl, Locally Causal Dynamical Triangulations in two dimensions, Phys. Rev. D 92 (2015) 084002 [arXiv:1507.04566, hep-th].

[67] J. Ambjørn, D.N. Coumbe, J. Gizbert-Studnicki and J. Jurkiewicz, Signature change of the metric in CDT quantum gravity?, JHEP 1508 (2015) 033 arXiv:1503.08580, hep-th].

[68] J. Ambjørn, J. Gizbert-Studnicki, A. Görlich, J. Jurkiewicz, N. Klitgaard and R. Loll, Characteristics of the new phase in CDT, Eur. Phys. J. C 77 (2017) 152 [arXiv:1610.05245, hep-th].

[69] J. Ambjørn, A. Görlich, S. Jordan, J. Jurkiewicz, and R. Loll, CDT meets Hořava-Lifshitz gravity, Phys. Lett. B 690 (2010) 413-419 arXiv:1002.3298, hep-th]. 
[70] J. Ambjørn, S. Jordan, J. Jurkiewicz and R. Loll, Quantum spacetime, from a practitioner's point of view, AIP Conf. Proc. 1514 (2012) 60 arXiv:1302.2181, hep-th].

[71] D.N. Coumbe, J. Gizbert-Studnicki and J. Jurkiewicz, Exploring the new phase transition of CDT, JHEP 1602 (2016) 144 [arXiv:1510.08672, hep-th].

[72] J. Ambjørn, J. Gizbert-Studnicki, A. Görlich and J. Jurkiewicz, The transfer matrix in four-dimensional CDT, JHEP 1209 (2012) 017 arXiv:1205.3791, hep-th].

[73] L. Bogacz, Z. Burda and B. Waclaw, Quantum widening of CDT universe, Phys. Rev. D 86 (2012) 104015 [arXiv:1204.1356, hep-th].

[74] J. Ambjørn, A. Görlich, J. Jurkiewicz, R. Loll, J. Gizbert-Studnicki and T. Trzesniewski, The semiclassical limit of Causal Dynamical Triangulations, Nucl. Phys. B 849 (2011) 144-165 [arXiv:1102.3929, hep-th].

[75] J. Gizbert-Studnicki, The effective action in four-dimensional CDT, $\mathrm{PhD}$ Thesis, Jagiellonian University Krakow, 2014 arXiv:1510.08719, hep-th].

[76] J. Ambjørn, J. Gizbert-Studnicki, A.T. Görlich, J. Jurkiewicz and R. Loll, The transfer matrix method in four-dimensional causal dynamical triangulations, AIP Conf. Proc. 1514 (2012) 67 [arXiv:1302.2210, hep-th].

[77] J. Ambjørn, J. Gizbert-Studnicki, A. Görlich and J. Jurkiewicz, The effective action in 4-dim CDT. The transfer matrix approach, JHEP 1406 (2014) 034 arXiv:1403.5940, hep-th].

[78] J. Ambjørn, A. Görlich, J. Jurkiewicz and R. Loll, CDT - an entropic theory of quantum gravity PoS CLAQG08 (2011) [arXiv:1007.2560, hep-th].

[79] J. Ambjørn, D. Coumbe, J. Gizbert-Studnicki and J. Jurkiewicz, Recent results in CDT quantum gravity, Proceedings of the 14th Marcel Grossmann Meeting, eds. M. Bianchi, R.T. Jantzen and R. Ruffini (World Scientific, Singapore, 2017) 3891-3896 [arXiv:1509.08788, gr-qc].

[80] J. Ambjørn, D. Coumbe, J. Gizbert-Studnicki, A. Görlich and J. Jurkiewicz, New higher-order transition in causal dynamical triangulations, Phys. Rev. D 95 (2017) 124029 arXiv:1704.04373, hep-lat].

[81] T. Hotta, T. Izubuchi and J. Nishimura, Singular vertices in the strong coupling phase of four-dimensional simplicial gravity, Nucl. Phys. Proc. Suppl. 47 (1996) 609-612 [arXiv: hep-lat/9511023]; S. Catterall, G. Thorleifsson, J.B. Kogut and R. Renken, Singular vertices and the triangulation space of the D sphere, Nucl. Phys. B 468 (1996) 263-276 [arXiv: hep-lat/9512012]. 
[82] J. Ambjørn, Z. Drogosz, J. Gizbert-Studnicki, A. Görlich, J. Jurkiewicz and D. Németh, Impact of topology in causal dynamical triangulations quantum gravity, Phys. Rev. D 94 (2016) 044010 [arXiv:1604.08786, hep-th].

[83] J. Ambjørn, J. Gizbert-Studnicki, A. Görlich, K. Grosvenor and J. Jurkiewicz, Four-dimensional CDT with toroidal topology, Nucl. Phys. B 922 (2017) 226-246 arXiv:1705.07653, hep-th].

[84] J. Ambjørn, J. Gizbert-Studnicki, A. Görlich, J. Jurkiewicz and D. Németh, The phase structure of Causal Dynamical Triangulations with toroidal spatial topology, JHEP 1806 (2018) 111 arXiv:1802.10434, hep-th].

[85] J. Ambjørn, A. Görlich, J. Jurkiewicz, A. Kreienbuehl and R. Loll, Renormalization group flow in CDT, Class. Quant. Grav. 31 (2014) 165003 arXiv:1405.4585, hep-th].

[86] I. Montvay and G. Münster, Quantum fields on a lattice (Cambridge University Press, Cambridge, 1994).

[87] J.H. Cooperman, On a renormalization group scheme for causal dynamical triangulations, Gen. Rel. Grav. 48 (2016) 29 [arXiv:1406.4531, gr-qc].

[88] J. Ambjørn, A. Görlich, J. Jurkiewicz and R. Loll, Causal Dynamical Triangulations and the search for a theory of quantum gravity, Proceedings of the 13th Marcel Grossmann Meeting, eds. K. Rosquist, R.T. Jantzen and R. Ruffini (World Scientific, Singapore, 2015) 120-137 arXiv:1305.6680, gr-qc].

[89] D.V. Vassilevich, Heat kernel expansion: user's manual, Phys. Rep. 388 (2003) 279-360 [arXiv: hep-th/0306138].

[90] D. Benedetti and J. Henson, Spectral geometry as a probe of quantum spacetime, Phys. Rev. D 80 (2009) 124036 [arXiv:0911.0401, hep-th].

[91] D.N. Coumbe and J. Jurkiewicz, Evidence for asymptotic safety from dimensional reduction in Causal Dynamical Triangulations, JHEP 1503 (2015) 151 arXiv:1411.7712, hep-th].

[92] J. Ambjørn, D. Coumbe, J. Gizbert-Studnicki and J. Jurkiewicz, Searching for a continuum limit in causal dynamical triangulation quantum gravity, Phys. Rev. D 93 (2016) 104032 arXiv:1603.02076, hep-th].

[93] J.H. Cooperman, Comments on "Searching for a continuum limit in CDT quantum gravity" arXiv:1604.01798, gr-qc]. 
[94] G. Calcagni, D. Oriti and J. Thürigen, Spectral dimension of quantum geometries, Class. Quant. Grav. 31 (2014) 135014 arXiv:1311.3340, hep-th].

[95] J. Ambjørn, A. Görlich, J. Jurkiewicz and R. Loll, Geometry of the quantum universe, Phys. Lett. B 690 (2010) 420-426 [arXiv:1001.4581, hep-th].

[96] G. Clemente, M. D'Elia and A. Ferraro, Spectral methods and running scales in Causal Dynamical Triangulations arXiv:1903.00430, hep-th].

[97] A. Görlich, Causal Dynamical Triangulations in four dimensions, PhD Thesis Jagiellonian University Krakow (2010) arXiv:1111.6938, hep-th].

[98] D.I. Diakonov and V.Yu. Petrov, Non-abelian Stokes theorems in the YangMills and gravity theories, J. Exp. Theor. Phys. 92 (2001) 905-920 [arXiv: hep-th/0008035].

[99] N. Klitgaard, R. Loll, M. Reitz and R. Toriumi: Geometric flux formula for the gravitational Wilson loop, to be published.

[100] N. Klitgaard and R. Loll, Introducing quantum Ricci curvature, Phys. Rev. D 97 (2018) 046008 [arXiv:1712.08847, hep-th].

[101] N. Klitgaard and R. Loll, Implementing quantum Ricci curvature, Phys. Rev. D 97 (2018) 106017 arXiv:1802.10524, hep-th].

[102] Y. Ollivier, Ricci curvature of Markov chains on metric spaces, J. Funct. Anal. 256 (2009) 810-864.

[103] N. Klitgaard and R. Loll, in preparation.

[104] L. Glaser and R. Loll, CDT and cosmology, Comptes Rendus Physique 18 (2017) 265-274 arXiv:1703.08160, gr-qc].

[105] T. Buchert, A.A. Coley, H. Kleinert, B.F. Roukema and D.L. Wiltshire, Observational challenges for the standard FLRW model, Int. J. Mod. Phys. D 25 (2016) 1630007 arXiv:1512.03313, astro-ph.CO].

[106] K. Bolejko and M. Korzynski, Inhomogeneous cosmology and backreaction: current status and future prospects, Int. J. Mod. Phys. D 26 (2017) 1730011 arXiv:1612.08222, gr-qc].

[107] J.H. Cooperman, Scale-dependent homogeneity measures for causal dynamical triangulations, Phys. Rev. D 90 (2014) 124053 [arXiv:1410.0632, gr-qc].

[108] K.G. Wilson, Confinement of quarks, Phys. Rev. D 10 (1974) 2445-2459. 